\title{
New Records of Spiders (Arachnida: Araneae) from Sinop Province, Turkey, Including an Annotated List of Species
}

\author{
Esra ETİRLI'1 ${ }^{1}$, Halil $\mathrm{KOÇ}^{* 1} 1^{\oplus}$, Zafer SANCAK ${ }^{2} \oplus$ \\ ${ }^{1}$ Sinop University, Department of Biology, Sinop, TURKEY \\ ${ }^{2}$ Kastamonu University, Department of Biology, Kastamonu 37100, TURKEY \\ *Corresponding author: koc.halil@hotmail.com
}

Received Date: 05.03.2018

Accepted Date: 31.12.2018

\section{Abstract}

Aim of study: This study was conducted to determine the biological richness of spiders from Sinop province, Turkey.

Area of study: The studies were carried out on between $41^{\circ} 12^{\prime}$ and $42^{\circ} 6^{\prime}$ northern latitudes and $34^{\circ}$ $14^{\prime}$ and $35^{\circ} 26^{\prime}$ east longitudes within the Sinop province borders.

Material and Methods: Spiders were collected from 2009 to 2015 using four collection methods: using sweep netting, tree beating, active searching and pitfall trapping.

Main results: A total of 1766 specimens were collected that represented by 31 families, 133 genera and 200 species. The most diverse families collected are the Salticidae (29 spp.), Theridiidae (29 spp.) and Gnaphosidae (21 spp.). According to previous studies and this work, 31 families, 139 genera, 212 species of spiders are present in Sinop province, of which 192 species are recorded for the first time.

Research highlights: Furthermore, one genus (Theridula Emerton 1882) and three species, namely Asagena meridionalis Kulczyński 1894, Theridion boesenbergi Strand 1904 and Theridula gonygaster (Simon 1873), are new records for Turkey, and are provided with illustrations. Here, we provide the first annotated checklist of the spider species of Sinop province.

Keywords: Species richness, Sinop, Araneae, Fauna, new records, checklist

\section{Detaylı Tür Listesini Içeren, Sinop İli Yeni Örümcek (Arachnida:}

\section{Araneae) Kayıtları}

$\ddot{O} \mathbf{z}$

Çalışmanin amacı: Bu çalışma, Sinop örümceklerinin biyolojik zenginliğini belirlemek amacıyla yapılmıştır.

Çalışma alanı: Çalışmalar, Sinop İl sınırları içinde, $41^{\circ} 12^{\prime}$ ve $42^{\circ} 6^{\prime}$ kuzey enlemleri ve $34^{\circ} 14^{\prime}$ ve $35^{\circ} 26^{\prime}$ doğu boylamlarında gerçekleştirilmiştir.

Materyal ve Yöntem: 2009-2015 yılları arasında yapılan arazi çalışmalarında örümcek örnekleri dört yöntem; atrap, dallara vurma, çevrede arama ve çukur tuzak kullanarak toplanmıştır.

Temel Sonuçlar: İncelenen toplam 1766 örneğin, 31 familya bağlı 133 cins ve 200 türe ait olduğu tespit edilmiştir. En çok dağılış gösteren familyalar sırasıyla; Salticidae (29 tür), Theridiidae (29 tür) and Gnaphosidae (21 tür)'dır. Daha önce yapılmış olan çalışmalar ve bu çalışma birlikte değerlendirildiğinde, 31 familya, 139 cins ve 212 türün Sinop’ta dağılış gösterdiği tespit edilmiştir. Bahsedilen bu türlerden 192 tür Sinop İl'i için yeni kayıttır.

Araştırma vurguları: Araştırma alanından bir cins (Theridula Emerton 1882) ve üç tür, Asagena meridionalis Kulczyński 1894, Theridion boesenbergi Strand 1904 and Theridula gonygaster (Simon 1873), Türkiye için yeni kayıttır. Ayrıca, bu çalışma ile Sinop'un örümcek türlerinin kontrol listesi ilk kez verilmektedir.

Anahtar Kelimeler: Tür zenginliği, Sinop, Araneae, fauna, yeni kayıt, kontrol listesi

\section{Introduction}

Currently, 4076 genera and 47297 spider species are classified in 116 families (World Spider Catalog, 2018). A large variety of spiders, are found in quite different terrestrial and fresh water habitats; from the peaks of
Everest to caves and houses (Bayram, 1987). Spiders are also hunted by other predators hunting for insects. They can reach 1000 individuals per square meter under suitable conditions. Spiders that are so dense in nature are thought to be able to stabilize 
insect populations. Therefore, their role in protecting the natural balance, biocontrol availability, and their nutritional ecology in agricultural ecosystems are being investigated thoroughly (Nyffeler and Benz, 1987).

Compared with the number of spiders known in the world, studies on spider systematics and fauna in Turkey are limited. Turkey's spider fauna was studied for the first time by Karol in 1967. Karol (1967) has published a Preliminary List of Spiders of Turkey I. According to this list, 302 species belonging to 119 genera, 30 families, are found in Turkey. In later years, Bayram (2002) and Demirsoy (2002) have contributed to the subject with the book "Zoogeography of Turkey". Accordingly, there are 162 genera and 520 species belonging to 44 families, and six subspecies in Turkey. Topçu, Demir \& Seyyar (2005) published a checklist of spiders of Turkey. They state that there are 613 species belonging to 43 families, and two subspecies. Later, 332 genera belonging to 53 families, 1022 species, were listed (Bayram, Kunt \& Danışman, 2017). The new list provided by Demir and Seyyar (2017) raises the total number of species known for Turkey to 1117 plus two subspecies, grouped in 339 genera and 52 families. However, there are no studies on the diversity of spiders in Sinop and the few previous records are due to sporadic collections. Brignoli (1978) reported 3 families, 5 genera and 5 species for Sinop. After that, Buchar and Dolanský (2011), Danışman, Erdek \& Coşar (2012a), Danışman, Erdek, M., Sancak, Z., Coşar (2012b,c) Danışman and Coşar (2016), Danışman (2014), Özkütük, Kunt, Karakaș \& Danışman, (2015), Coşar (2015), Coșar and Varol (2016), Coşar, Danışman \& Tarlabölen (2016), Coşar, Danışman \& Kartaler (2017) and Logunov and Demir (2006) found new records for 14 species of 12 genera of 8 families. Until 2018, 11 families, 17 genera and 19 species have been reported from Sinop province, which is consistent with the above information.

The aim of this study is to contribute to the spider fauna of the Central Black Sea Region and Turkey, which is not very well investigated. Moreover, a preliminary checklist for Sinop province is provided.

\section{Material and Methods \\ Study Area}

Sinop is located between $41^{\circ} 12^{\prime}$ and $42^{\circ}$ $6^{\prime}$ northern latitudes and $34^{\circ} 14^{\prime}$ and $35^{\circ} 26^{\prime}$ east longitudes, in the region where the Eastern and Western Black Sea climates are intertwined. In the Black Sea Region, the sea increases the humidity of the region and does not allow the increase of temperature differences in the coastal regions. However, because of their highness, the climatic characteristics of the mountains facing north and south are different. Due to the mountains running parallel to the coast in the province of Sinop, there is no maritime climate. The climate in the coastal zone is moderate. The climate changes in distant districts, such as Boyabat, Durağan and Saraydüzü. In these districts, a transition climate zone is observed between the Black Sea climate and the continental climate of Central Anatolia (Anonymous, 2012).

According to the 14-year observations obtained from the Sinop Meteorology Station Directorate, average annual temperature is $14.8^{\circ} \mathrm{C}$ and average annual precipitation is $64.1 \mathrm{~mm}$.

The natural vegetation is rich and diverse because the Black Sea climate is rainy throughout the year. The soil structure is very suitable for the growth of woody herbs since it has a stony, clayey and calcareous sandy character. There are forest, maquis frigana, sand dune, stream, marsh and lake vegetation types. The natural vegetation covers the forests. Climate transition is also evident in the floristic structure. Mediterranean vegetation is also found along the coastline of the province; the climate starts to become dry towards the south. For this reason, the lush vegetation cover in the north replaces steppe plants. The main tree species on the coastline of the province are pine, beech, hornbeam, oak, linden, plane-tree, poplar and chestnut. As the altitude increases, yellow pine, fir, ash, and elm trees are found. Due to higher in the forest understory, plant density on the forest floor is higher (Baysal, 2008; Öztürk, 2010). 


\section{Material}

Spiders were collected from 67 different localities. A total of 1766 adult specimens were collected from Sinop and its vicinity (Fig. 1) and 31 families were found. The study localities, with their coordinates, elevation and sampling dates, are numbered and given in Table 1.

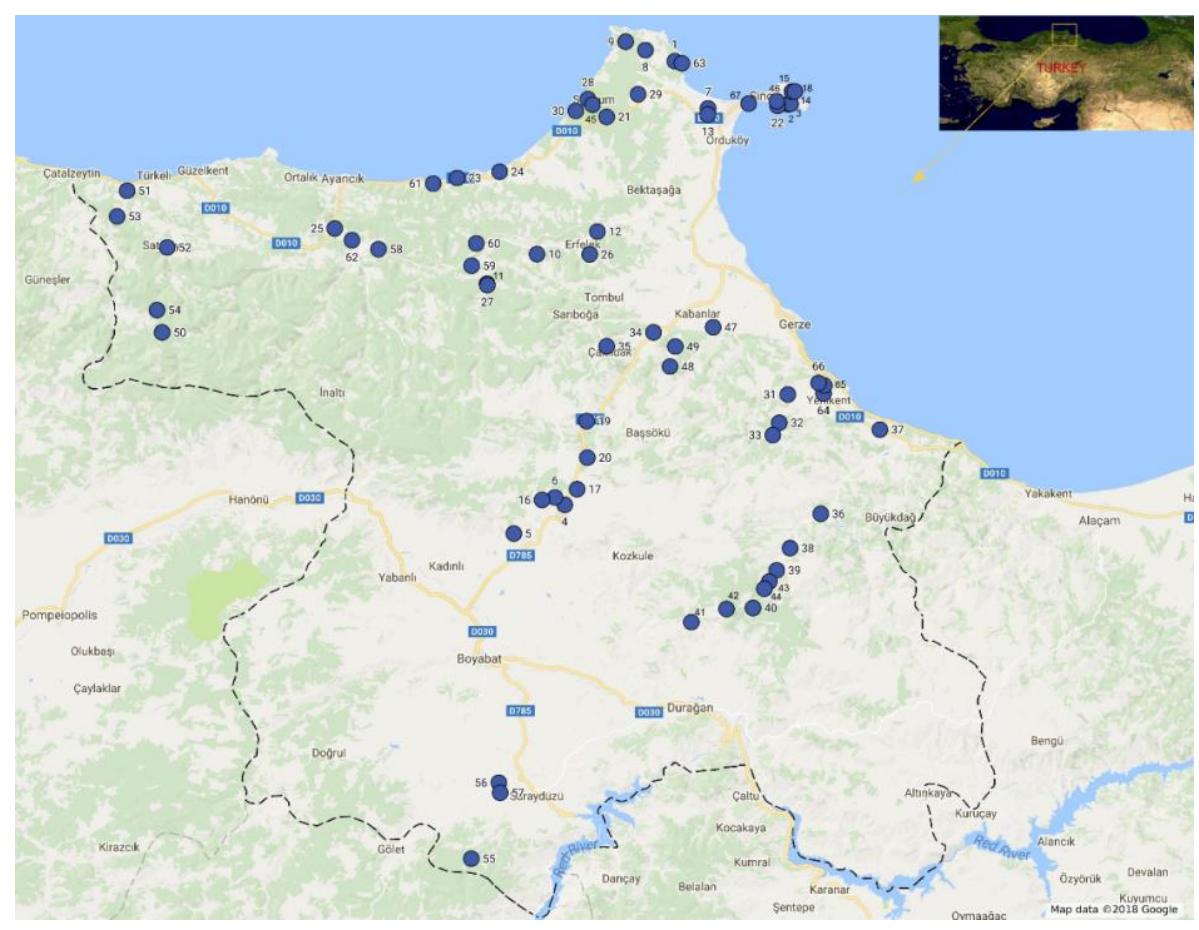

Figure 1. Map of Sinop Province and its environs; numbered localities are presented in Table 1. 


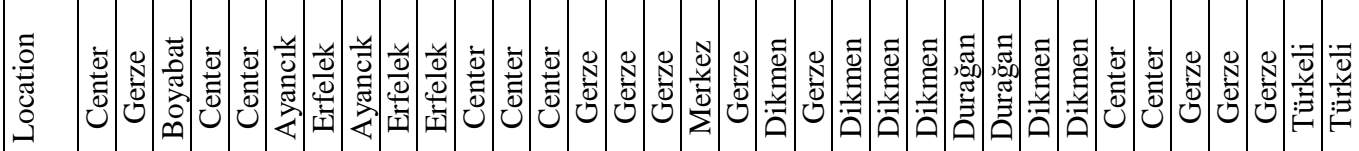

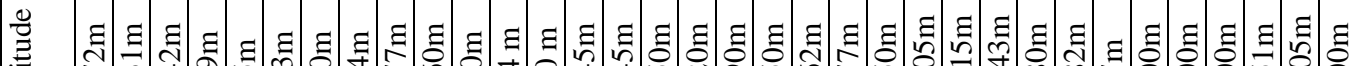

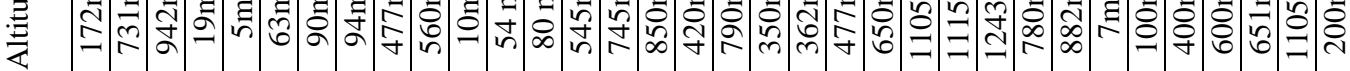

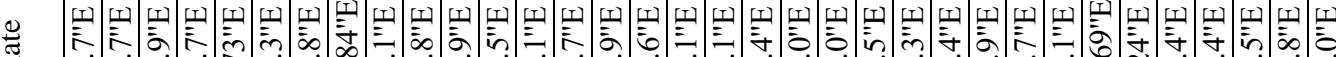

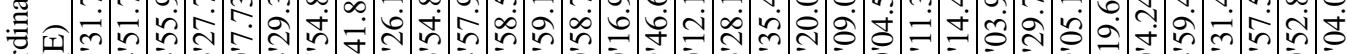

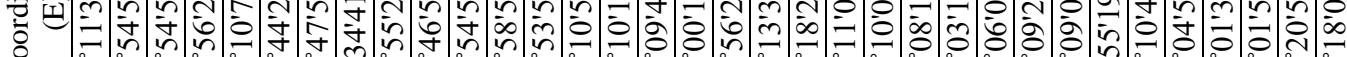

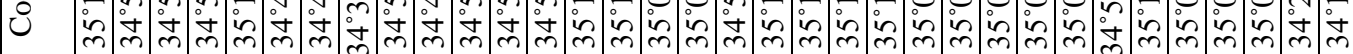

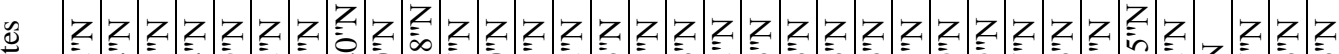
茜

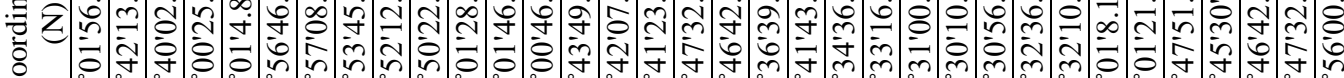

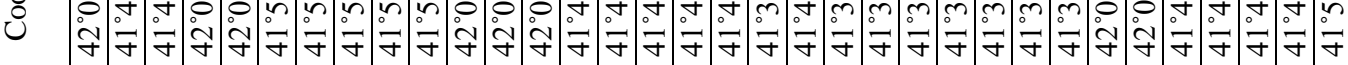

•

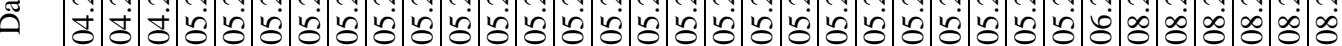

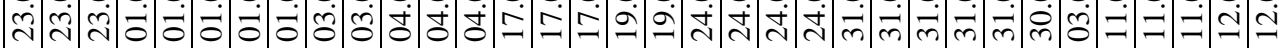

产

రั)

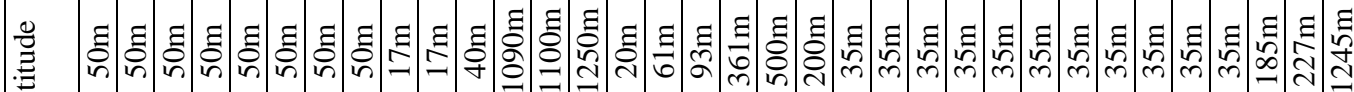
Uे

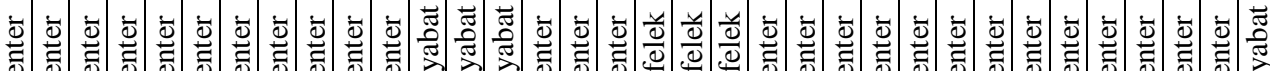

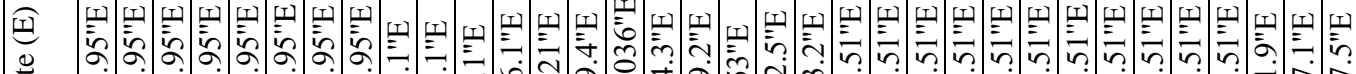

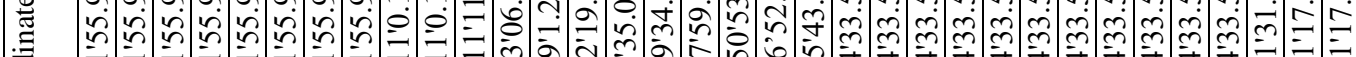
:

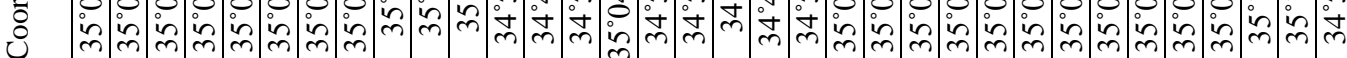

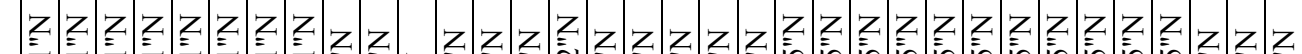
盖

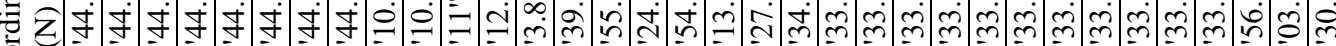

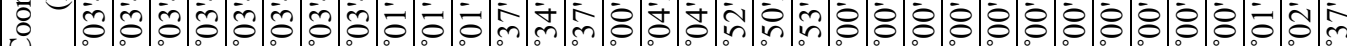
F)

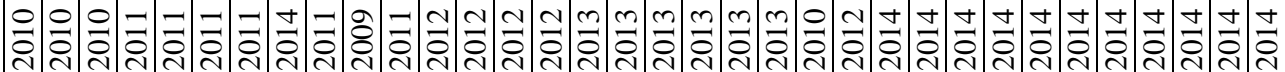

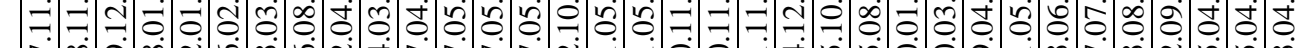

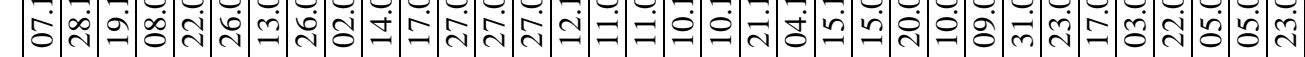




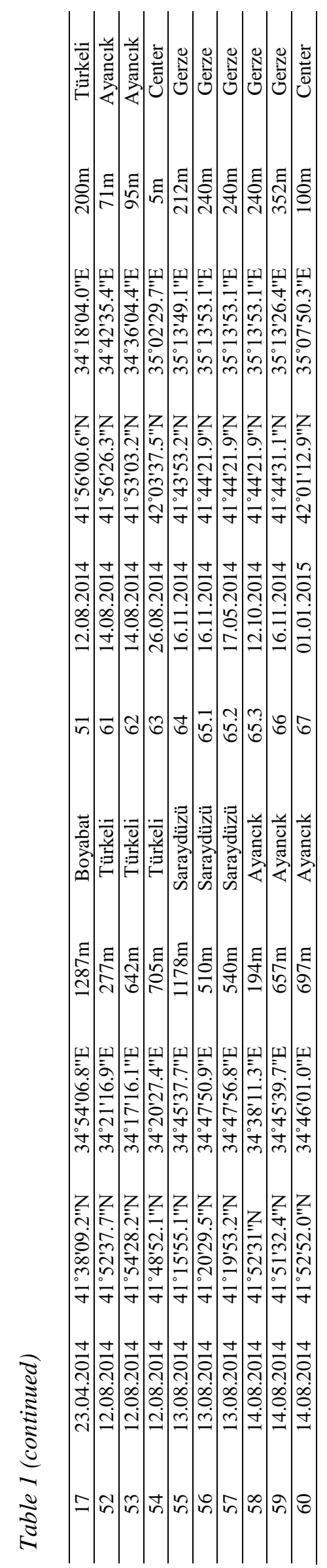


We used hand collecting, soil and litter sampling, beating and/or sweeping vegetation, and pitfall trapping to collect spiders from rocks (underneath), crevices, logs and debris; under loose bark of dead trees, stumps and the forest understory.

Captured spiders were transferred by aspirator to small jars or vials containing $70 \%$ ethyl alcohol. In the laboratory, all spiders were sorted, counted and identified using appropriate literature. All collected spiders were taken to the laboratory, placed in small dishes, submerged in alcohol, and examined using stereo-zoom microscopes.

We used the identification keys of Nentwig, Blick, Gloor, Hänggi \& Kropf (2017), and followed the World Spider Catalog (2018) for the nomenclature and taxonomy of spiders. Voucher specimens were stored in $70 \%$ ethanol and deposited in the Department of Biology, Faculty of Science and Art, Sinop University, Sinop. Specimens were photographed using a Canon
EOS 70D camera attached to a Zeiss Stemi 2000-C stereomicroscope.

For consistency purposes, we follow the currently accepted names, as listed in the World Spider Catalog (2018). Family order is based on the database prepared by Bayram, Kunt \& Danışman (2017). The species name and genus are sorted in alphabetical order.

\section{Results and discussion}

Spider families, species and locality numbers are listed in order for convenience. After each listed species, the distribution by realm (Palaearctic, Holarctic, Cosmopolitan etc.) is given, based chiefly on the designations (Table 2). In addition, the number of male and female individuals collected for each species and the total number of individuals caught by family members are also included in Table 2. Subadult individuals are not included in this list.

Table 2. Families and species, distribution, localities and number of male and female spiders collected in Sinop

\section{Family: SCYTODIDAE}

\begin{tabular}{cllcc} 
Taxon & \multicolumn{1}{c}{ Distribution } & Locality & \multirow{1}{c}{} & + \\
\hline $\begin{array}{c}\text { Scytodes thoracica } \\
\text { (Latreille 1802) }\end{array}$ & Holarctic, Pasific & {$[1.1][5][30][39]$} & 3 & 9 \\
& & {$[38][37][47]$} & & \\
\cline { 2 - 4 } & & Total & 12
\end{tabular}

Family: PHOLCIDAE

\begin{tabular}{|c|c|c|c|c|}
\hline Taxon & Distribution & Locality & o & q \\
\hline $\begin{array}{l}\text { Holocnemus pluchei } \\
\text { (Scopoli 1763) }\end{array}$ & Europe, Northern Africa & [1.2] [51] & 2 & 2 \\
\hline \multirow[t]{2}{*}{$\begin{array}{l}\text { Pholcus phalangioides } \\
\quad \text { (Fuesslin 1775) }\end{array}$} & Cosmopolitan & {$[13.7][67]$} & 1 & 1 \\
\hline & & Total & & 5 \\
\hline
\end{tabular}

Family:

SEGESTRIIDAE

\begin{tabular}{cllcc}
\multicolumn{1}{c}{ Taxon } & \multicolumn{1}{c}{ Distribution } & Locality & \multirow{1}{c}{} & + \\
\hline $\begin{array}{c}\text { Segestria florentina } \\
\text { (Rossi 1790) }\end{array}$ & $\begin{array}{l}\text { European to Georgia, } \\
\text { Brazil, Uruguay, } \\
\text { Argentina }\end{array}$ & {$[46]$} & 0 & 1 \\
$\begin{array}{c}\text { Segestria senoculata } \\
\text { (Linnaeus 1758) }\end{array}$ & Palearctic & [42] [53] [55] [62] & 0 & 4 \\
\cline { 2 - 5 } & & Total & 5
\end{tabular}


Table 2 (continued)

Family: DYSDERIDAE

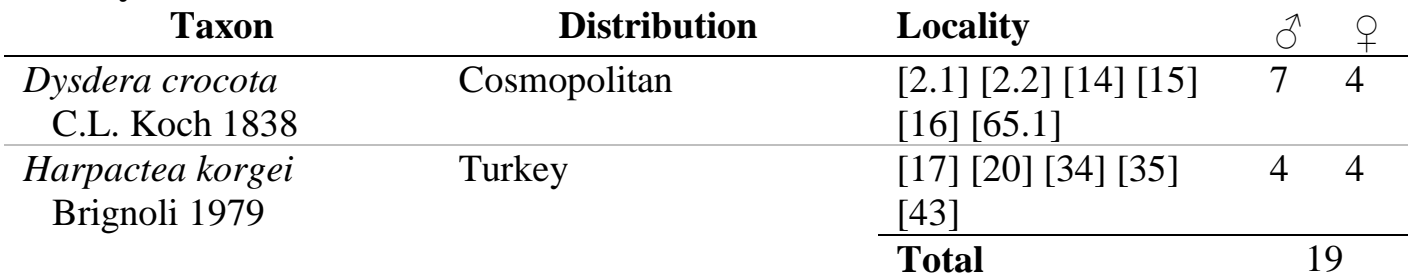

Family: MIMETIDAE

\begin{tabular}{|c|c|c|c|c|}
\hline Taxon & Distribution & Locality & $\hat{0}$ & 우 \\
\hline $\begin{array}{l}\text { Ero aphana } \\
\quad \text { (Walckenaer 1802) }\end{array}$ & Palearctic & {$[13.8]$} & 0 & 1 \\
\hline \multirow{2}{*}{$\begin{array}{l}\text { Mimetus laevigatus } \\
\quad \text { (Keyserling 1863) }\end{array}$} & Mediterranean-Asian & [37] & 0 & 3 \\
\hline & & Total & & \\
\hline
\end{tabular}

Family: OECOBIIDAE

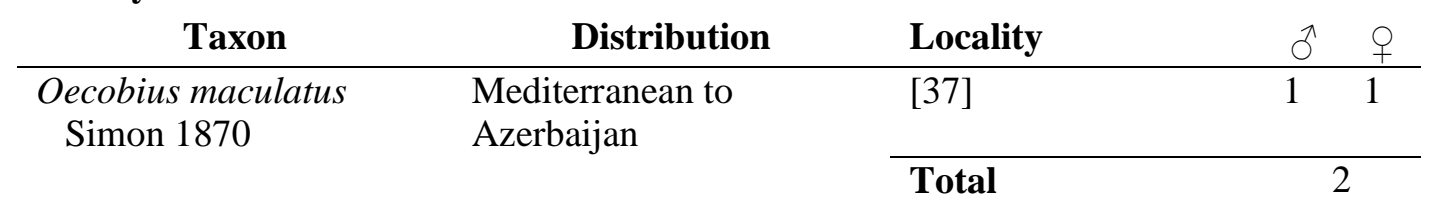

Family: ULOBORIDAE

\begin{tabular}{cllcc}
\multicolumn{1}{c}{ Taxon } & \multicolumn{1}{c}{ Distribution } & Locality & \multirow{2}{c}{} & \multicolumn{1}{c}{} \\
\hline Hyptiotes paradoxus & European, Turkey, & {$[61][11][41][59]$} & 8 & 3 \\
(C.L. Koch 1834) & Caucasia & {$[60][65.3]$} & & \\
$\begin{array}{c}\text { Uloborus walckenaerius } \\
\text { Latreille 1806 }\end{array}$ & Palearctic & {$[57][58]$} & 1 & 2 \\
\cline { 2 - 5 } & & Total & &
\end{tabular}

Family: THERIDIIDAE

\begin{tabular}{|c|c|c|c|c|}
\hline Taxon & Distribution & Locality & $0^{\pi}$ & 오 \\
\hline $\begin{array}{l}\text { Anelosimus vittatus } \\
\text { (C.L. Koch 1836) }\end{array}$ & Palearctic & $\begin{array}{l}{[23][25][28][30]} \\
{[33][36][37][38]} \\
{[43][39][44][47]} \\
{[59][60][65.2]}\end{array}$ & 13 & 43 \\
\hline $\begin{array}{l}\text { *Asagena meridionalis } \\
\text { Kulczyński } 1894\end{array}$ & East European to Georgia & [4] & 1 & 0 \\
\hline $\begin{array}{l}\text { Asagena phalerata } \\
\text { (Panzer 1801) }\end{array}$ & Palearctic & [16] [23] & 1 & 1 \\
\hline $\begin{array}{l}\text { Crustulina guttata } \\
\text { (Wider 1834) }\end{array}$ & Palearctic & {$[10][15][30][38]$} & 1 & 3 \\
\hline $\begin{array}{l}\text { Dipoena melanogaster } \\
\text { (C.L. Koch 1837) }\end{array}$ & $\begin{array}{l}\text { European, } \\
\text { North Africa to } \\
\text { Azerbaijan, Iran }\end{array}$ & {$[36][65.2]$} & 2 & 1 \\
\hline $\begin{array}{l}\text { Enoplognatha ovata } \\
\quad \text { (Clerck 1757) }\end{array}$ & Holarctic & {$[62][53]$} & 0 & 2 \\
\hline $\begin{array}{l}\text { Enoplognatha thoracica } \\
\text { (Hahn 1833) }\end{array}$ & Holarctic & [4] & 1 & 0 \\
\hline $\begin{array}{l}\text { Episinus maculipes } \\
\text { Cavanna } 1876\end{array}$ & $\begin{array}{l}\text { Southern Europe, } \\
\text { Algeria, Turkey }\end{array}$ & [53] & 1 & 0 \\
\hline
\end{tabular}


Table 2 (continued)

\begin{tabular}{|c|c|c|c|c|}
\hline $\begin{array}{l}\text { Episinus truncatus } \\
\text { Latreille } 1809\end{array}$ & $\begin{array}{l}\text { Europe, Turkey, } \\
\text { Caucasus }\end{array}$ & [53] & 0 & 1 \\
\hline $\begin{array}{l}\text { Euryopis flavomaculata } \\
\text { (C.L. Koch 1836) }\end{array}$ & Palearctic & [42] & 1 & 0 \\
\hline $\begin{array}{l}\text { Heterotheridion } \\
\text { nigrovariegatum } \\
\text { (Simon, 1873) }\end{array}$ & Palearctic & [49] [53] [55] & 0 & 3 \\
\hline $\begin{array}{l}\text { Kochiura aulica } \\
\quad \text { (C.L. Koch 1838) }\end{array}$ & $\begin{array}{l}\text { Canarian Islands, Cape } \\
\text { Verde Islands to } \\
\text { Azerbaijan }\end{array}$ & $\begin{array}{l}{[28][37][65.1]} \\
{[65.2][65.3]}\end{array}$ & 10 & 9 \\
\hline $\begin{array}{l}\text { Lasaeola tristis } \\
\text { (Hahn 1833) }\end{array}$ & European to Middle Asia & [44] & 2 & 0 \\
\hline $\begin{array}{l}\text { Neottiura bimaculata } \\
\text { Linnaeus } 1767\end{array}$ & Holarctic & [44] & 1 & 0 \\
\hline $\begin{array}{l}\text { Parasteatoda lunata } \\
\text { (Clerck 1757) }\end{array}$ & Palearctic & {$[45][63]$} & 0 & 4 \\
\hline $\begin{array}{l}\text { Parasteatoda } \\
\text { tepidariorum } \\
\text { (C.L. Koch 1841) }\end{array}$ & Cosmopolitan & $\begin{array}{l}{[7][13.5][13.7]} \\
{[13.8]}\end{array}$ & 14 & 24 \\
\hline $\begin{array}{l}\text { Phycosoma inornatum } \\
\text { (O.P.-Cambridge 1861) }\end{array}$ & European to Middle Asia & [27] & 1 & 0 \\
\hline $\begin{array}{l}\text { Platnickina tincta } \\
\text { (Walckenaer 1802) }\end{array}$ & Holarctic & $\begin{array}{l}{[27][36][38][39]} \\
{[42][43][44][47]} \\
{[63]}\end{array}$ & 7 & 14 \\
\hline $\begin{array}{l}\text { Robertus arundineti } \\
\text { (O.P.-Cambridge 1871) }\end{array}$ & Palearctic & {$[24][42][50]$} & 1 & 4 \\
\hline $\begin{array}{l}\text { Simitidion simile } \\
\quad \text { (C.L. Koch 1836) }\end{array}$ & Holarctic & [34] [44] [51] [65.2] & 1 & 6 \\
\hline $\begin{array}{l}\text { Steatoda albomaculata } \\
\text { (De Geer 1778) }\end{array}$ & Cosmopolitan & [37] & 0 & 1 \\
\hline $\begin{array}{l}\text { Steatoda bipunctata } \\
\text { (Linnaeus 1758) }\end{array}$ & Holarctic & [35] & 0 & 1 \\
\hline $\begin{array}{l}\text { Steatoda grossa } \\
\text { (C.L. Koch 1838) }\end{array}$ & Cosmopolitan & {$[13.2]$} & 1 & 0 \\
\hline $\begin{array}{r}\text { Steatoda paykulliana } \\
\text { (Walckenaer 1805) }\end{array}$ & $\begin{array}{l}\text { European, Mediterranean } \\
\text { to Middle Asia }\end{array}$ & $\begin{array}{l}{[2.1][2.2][4][13.4]} \\
{[15][19][34][65.3]}\end{array}$ & 3 & 6 \\
\hline $\begin{array}{l}\text { Steatoda triangulosa } \\
\text { (Walckenaer 1802) }\end{array}$ & Cosmopolitan & [7] [13.1] [13.8] & 3 & 6 \\
\hline $\begin{array}{l}\text { *Theridion boesenbergi } \\
\text { Strand } 1904\end{array}$ & European, Caucasia & [43] & 0 & 1 \\
\hline $\begin{array}{l}\text { Theridion melanurum } \\
\text { Hahn } 1831\end{array}$ & Holarctic, Azor & {$[65.2]$} & 1 & 0 \\
\hline $\begin{array}{l}\text { Theridion varians } \\
\text { Hahn } 1833\end{array}$ & Holarctic & $\begin{array}{l}{[26][27][29][30]} \\
{[50][53][63]}\end{array}$ & 2 & 5 \\
\hline \multirow[t]{2}{*}{$\begin{array}{l}\text { *Theridula gonygaster } \\
\quad \text { (Simon 1873) }\end{array}$} & Cosmopolitan & {$[10]$} & 0 & 1 \\
\hline & & Total & & \\
\hline
\end{tabular}


Table 2 (continued)

Family:

LINYPHIIDAE

\begin{tabular}{|c|c|c|c|c|}
\hline Taxon & Distribution & Locality & $0^{\pi}$ & 우 \\
\hline $\begin{array}{l}\text { Agyneta rurestris } \\
\text { (C.L. Koch 1836) }\end{array}$ & Palearctic & {$[65.3]$} & 1 & 0 \\
\hline $\begin{array}{l}\text { Centromerus albidus } \\
\text { Simon } 1929\end{array}$ & European, Turkey & [17] [26] [30] [43] & 0 & 9 \\
\hline $\begin{array}{l}\text { Centromerus sylvaticus } \\
\text { (Blackwall 1841) }\end{array}$ & Holarctic & {$[1.6][1.7]$} & 4 & 0 \\
\hline $\begin{array}{l}\text { Cresmatoneta mutinensis } \\
\text { (Canestrini 1868) }\end{array}$ & Palearctic & {$[13.4]$} & 0 & 1 \\
\hline $\begin{array}{l}\text { Erigone dentipalpis } \\
\text { (Wider 1834) }\end{array}$ & Holarctic & [25] & 1 & 0 \\
\hline $\begin{array}{l}\text { Erigonoplus } \\
\text { spinifemuralis } \\
\text { Dimitrov } 2003 \\
\end{array}$ & $\begin{array}{l}\text { Greece, Crete, } \\
\text { Macedonia, Bulgaria, } \\
\text { Turkey, Ukraine }\end{array}$ & [16] & 1 & 0 \\
\hline $\begin{array}{l}\text { Frontinellina frutetorum } \\
\text { (C.L. Koch 1834) }\end{array}$ & Palearctic & $\begin{array}{l}5][23][24][32] \\
{[34][35][36][37]} \\
{[39][42][44][47]} \\
{[48][52][57][59]} \\
{[65.2]}\end{array}$ & 22 & 62 \\
\hline $\begin{array}{l}\text { Gnathonarium dentatum } \\
\text { (Wider 1834) }\end{array}$ & Palearctic & {$[45]$} & 1 & 0 \\
\hline $\begin{array}{l}\text { Lepthyphantes leprosus } \\
\text { (Ohlert 1865) }\end{array}$ & Holarctic, Chile & [59] & 0 & 1 \\
\hline $\begin{array}{l}\text { Linyphia hortensis } \\
\text { Sundevall } 1830\end{array}$ & Palearctic & [42] & 1 & 7 \\
\hline $\begin{array}{l}\text { Linyphia triangularis } \\
\text { (Clerck 1757) }\end{array}$ & Palearctic, USA & $\begin{array}{l}{[47][49][53][54]} \\
{[59]}\end{array}$ & 15 & 0 \\
\hline $\begin{array}{l}\text { Neriene furtiva } \\
\text { (O. P.-Cambridge } \\
\text { 1871) }\end{array}$ & $\begin{array}{l}\text { European, North Africa, } \\
\text { Russia, Ukraine }\end{array}$ & {$[1.2]$} & 1 & 0 \\
\hline $\begin{array}{r}\text { Neriene peltata } \\
\text { (Wider 1834) }\end{array}$ & Palearctic, Greenland & $\begin{array}{l}{[20][27][37][36]} \\
{[43]}\end{array}$ & 0 & 6 \\
\hline $\begin{array}{l}\text { Neriene radiata } \\
\text { (Walckenaer } 1841)\end{array}$ & Holarctic & $\begin{array}{l}{[8][21][27][40]} \\
{[61]}\end{array}$ & 8 & 29 \\
\hline $\begin{array}{l}\text { Oedothorax gibbosus } \\
\text { (Blackwall 1841) }\end{array}$ & Palearctic & {$[45]$} & 0 & 1 \\
\hline $\begin{array}{l}\text { Palliduphantes khobarum } \\
\text { (Charitonov 1947) }\end{array}$ & $\begin{array}{l}\text { Greece, Turkey, Ukraine, } \\
\text { Russia, Middle Asia }\end{array}$ & $\begin{array}{l}{[1.2 .][1.4][1.5][1.6]} \\
{[1.7][33][40][42]}\end{array}$ & 8 & 11 \\
\hline $\begin{array}{l}\text { Prinerigone vagans } \\
\text { (Audouin } 1826 \text { ) }\end{array}$ & Old World & {$[28][44]$} & 2 & 0 \\
\hline $\begin{array}{l}\text { Stemonyphantes } \\
\text { montanus } \\
\text { Wunderlich } 1978\end{array}$ & Turkey & $\begin{array}{l}{[1.1][1.2][1.3][1.5]} \\
{[2.2][10][20][50]}\end{array}$ & 4 & 5 \\
\hline $\begin{array}{l}\text { Tenuiphantes flavipes } \\
\text { (Blackwall,1854) }\end{array}$ & European & $\begin{array}{l}{[1.5][1.2][11][24]} \\
{[26][27][31][39]} \\
{[43][50][65.1]}\end{array}$ & 2 & 31 \\
\hline
\end{tabular}


Table 2 (continued)

\begin{tabular}{|c|c|c|c|}
\hline $\begin{array}{l}\text { Trematocephalus } \\
\text { cristatus } \\
\quad \text { (Wider 1834) }\end{array}$ & Palearctic & [21] [27] & 2 \\
\hline
\end{tabular}

\begin{tabular}{|c|c|c|c|c|}
\hline \multirow{2}{*}{$\begin{array}{l}\text { Family: } \\
\text { TETRAGNATHIDAE }\end{array}$} & & \multirow[t]{2}{*}{ Total } & \multicolumn{2}{|c|}{237} \\
\hline & & & & \\
\hline Taxon & Distribution & Locality & $\sigma^{\pi}$ & q \\
\hline $\begin{array}{l}\text { Metellina mengei } \\
\text { (Blackwall 1869) }\end{array}$ & $\begin{array}{l}\text { European to Caucasia, } \\
\text { Altay, Iran }\end{array}$ & [10] [21] [27] [35] & 7 & 11 \\
\hline $\begin{array}{l}\text { Metellina segmentata } \\
\quad \text { (Clerck 1757) }\end{array}$ & Palearctic & $\begin{array}{l}{[10][11][12][26]} \\
{[28][42][43][47]} \\
{[60][65.1][66]}\end{array}$ & 16 & 17 \\
\hline $\begin{array}{l}\text { Tetragnatha extensa } \\
\text { (Linnaeus 1758) }\end{array}$ & Holarctic, Madeira & {$[65.2]$} & 1 & 0 \\
\hline $\begin{array}{l}\text { Tetragnatha montana } \\
\text { Simon } 1874\end{array}$ & Palearctic & [8] [24] [39] & 2 & 1 \\
\hline $\begin{array}{c}\text { Tetragnatha obtusa } \\
\text { C.L. Koch } 1837\end{array}$ & Palearctic & $\begin{array}{l}{[11][24][30][36]} \\
{[43][52][54][55]}\end{array}$ & 1 & 9 \\
\hline & & Total & & 5 \\
\hline
\end{tabular}

\begin{tabular}{|c|c|c|c|c|}
\hline Taxon & Distribution & Locality & $\hat{0}$ & q \\
\hline $\begin{array}{l}\text { Agalenatea redii } \\
\text { (Scopoli 1763) }\end{array}$ & Palearctic & {$[18][65.2]$} & 1 & 2 \\
\hline $\begin{array}{l}\text { Araneus angulatus } \\
\text { Clerck } 1757\end{array}$ & Palearctic & $\begin{array}{l}{[1.8][13.9][13.10]} \\
{[59]}\end{array}$ & 0 & 4 \\
\hline $\begin{array}{l}\text { Araneus diadematus } \\
\text { Clerck } 1757\end{array}$ & Holarctic & {$[10][11]$} & 0 & 5 \\
\hline $\begin{array}{l}\text { Araneus sturmi } \\
(\text { Hahn 1831) }\end{array}$ & Palearctic & [43] & 0 & 1 \\
\hline $\begin{array}{l}\text { Araniella cucurbitina } \\
\text { (Clerck 1757) }\end{array}$ & Palearctic & $\begin{array}{l}{[23][34][37][38]} \\
{[39][42][44]}\end{array}$ & 7 & 14 \\
\hline $\begin{array}{l}\text { Argiope bruennichi } \\
\text { (Scopoli 1772) }\end{array}$ & Palearctic & $\begin{array}{l}{[13.10][48][57]} \\
{[65.3]}\end{array}$ & 2 & 12 \\
\hline $\begin{array}{l}\text { Argiope lobata } \\
\text { (Pallas 1772) }\end{array}$ & Old World & [56] [57] & 2 & 4 \\
\hline $\begin{array}{l}\text { Cyclosa conica } \\
\text { (Pallas 1772) }\end{array}$ & Holarctic & [5] [8] [23] [27] [34] & 2 & 4 \\
\hline $\begin{array}{l}\text { Cyrtarachne ixoides } \\
\text { (Simon 1870) }\end{array}$ & $\begin{array}{l}\text { Mediterranean to } \\
\text { Georgia, Madagascar }\end{array}$ & [45] & 0 & 1 \\
\hline $\begin{array}{l}\text { Gibbaranea } \\
\text { bituberculata } \\
\quad \text { (Walckenaer 1802) }\end{array}$ & Palearctic & [21] & 0 & 1 \\
\hline $\begin{array}{l}\text { Hypsosing a albovittata } \\
\text { (Westring 1851) }\end{array}$ & Palearctic & [4] [6] & 2 & 0 \\
\hline $\begin{array}{l}\text { Hypsosinga pygmaea } \\
\text { (Sundevall 1831) }\end{array}$ & Holarctic & [13.11] [47] & 1 & 1 \\
\hline $\begin{array}{l}\text { Hypsosinga sanguinea } \\
\text { (C.L. Koch 1844) }\end{array}$ & Palearctic & {$[32]$} & 1 & 1 \\
\hline
\end{tabular}


Table 2 (continued)

\begin{tabular}{|c|c|c|c|c|}
\hline $\begin{array}{l}\text { Larinioides cornutus } \\
\text { (Clerck 1757) }\end{array}$ & Holarctic & [13.4] [13.6] [13.9] & 2 & 4 \\
\hline $\begin{array}{l}\text { Larinioides suspicax } \\
\text { (O. P.-Cambridge, } \\
\text { 1876) }\end{array}$ & $\begin{array}{l}\text { European, North Africa } \\
\text { to Middle Asia }\end{array}$ & [7] [13.5] & 0 & 4 \\
\hline $\begin{array}{l}\text { Mangora acalypha } \\
\text { (Walckenaer 1802) }\end{array}$ & Palearctic & $\begin{array}{l}{[8][23][24][28]} \\
{[32][34][35][36]} \\
{[37][38][39][44]} \\
{[42][51][54][59]} \\
{[62][65.2]}\end{array}$ & 53 & 61 \\
\hline $\begin{array}{l}\text { Neoscona adianta } \\
\text { (Walckenaer 1802) }\end{array}$ & Palearctic & [7] [13.10] [47] & 0 & 7 \\
\hline $\begin{array}{l}\text { Neoscona subfusca } \\
\text { (C.L. Koch 1837) }\end{array}$ & Old World & [45] [56] [58] [62] & 0 & 4 \\
\hline $\begin{array}{l}\text { Nuctenea umbratica } \\
\text { (Clerck 1757) }\end{array}$ & European to Azerbaijan & [13.7] [42] & 1 & 1 \\
\hline $\begin{array}{l}\text { Zilla diodia } \\
\text { (Walckenaer 1802) }\end{array}$ & European to Azerbaijan & $\begin{array}{l}{[8][9][23][24][25]} \\
{[26][27][28][30]} \\
{[31][37][51][65.2]}\end{array}$ & 16 & 35 \\
\hline & & Total & 256 & \\
\hline \multicolumn{5}{|l|}{ Family: LYCOSIDAE } \\
\hline Taxon & Distribution & Locality & $\widehat{0}$ & q \\
\hline $\begin{array}{l}\text { Alopecosa farinosa } \\
\text { (Herman 1879) }\end{array}$ & Palearctic & $\begin{array}{l}{[13.5][16][17]} \\
{[65.2]}\end{array}$ & 3 & 11 \\
\hline $\begin{array}{l}\text { Alopecosa albofasciata } \\
\text { (Brullé 1832) }\end{array}$ & $\begin{array}{l}\text { Mediterranean to Middle } \\
\text { Asia }\end{array}$ & $\begin{array}{l}{[8][9][13.5][18]} \\
{[22][23][28][34]} \\
{[36][37][38][45]} \\
{[65.2]}\end{array}$ & 9 & 30 \\
\hline $\begin{array}{l}\text { Alopecosa cursor } \\
\text { (Hahn 1831) }\end{array}$ & Palearctic & {$[17]$} & 0 & 2 \\
\hline $\begin{array}{l}\text { Alopecosa pulverulenta } \\
\text { (Clerck 1757) }\end{array}$ & Palearctic & $\begin{array}{l}{[16][19][23][26]} \\
{[28][32][42]}\end{array}$ & 6 & 6 \\
\hline $\begin{array}{l}\text { Aulonia albimana } \\
\text { (Walckenaer 1805) }\end{array}$ & Palearctic & $\begin{array}{l}{[23][32][34][36]} \\
{[38][41][42]}\end{array}$ & 4 & 7 \\
\hline $\begin{array}{l}\text { Hogna radiata } \\
\text { (Latreille 1817) }\end{array}$ & $\begin{array}{l}\text { Middle European to } \\
\text { Middle Asia, Iran, } \\
\text { Middle Africa }\end{array}$ & {$[13.11][48]$} & 1 & 2 \\
\hline $\begin{array}{l}\text { Pardosa hortensis } \\
\text { (Thorell 1872) }\end{array}$ & Palearctic & $\begin{array}{l}8][9][13.5][16] \\
{[17][23][24][26]} \\
{[42][43]}\end{array}$ & 6 & 19 \\
\hline $\begin{array}{l}\text { Pardosa lugubris } \\
\text { (Walckenaer 1802) }\end{array}$ & $\begin{array}{l}\text { Western and Central } \\
\text { Palearctic }\end{array}$ & $\begin{array}{l}{[8][17][20][26]} \\
{[55][42]}\end{array}$ & 23 & 11 \\
\hline $\begin{array}{l}\text { Trochosa hispanica } \\
\text { Simon } 1870\end{array}$ & $\begin{array}{l}\text { Mediterranean to Middle } \\
\text { Asia, Iran }\end{array}$ & [8] [13.4] [28] [39] & 0 & 5 \\
\hline $\begin{array}{l}\text { Trochosa terricola } \\
\text { Thorell } 1856\end{array}$ & Holarctic & {$[2.1][13.5]$} & 2 & 1 \\
\hline
\end{tabular}


Table 2 (continued)

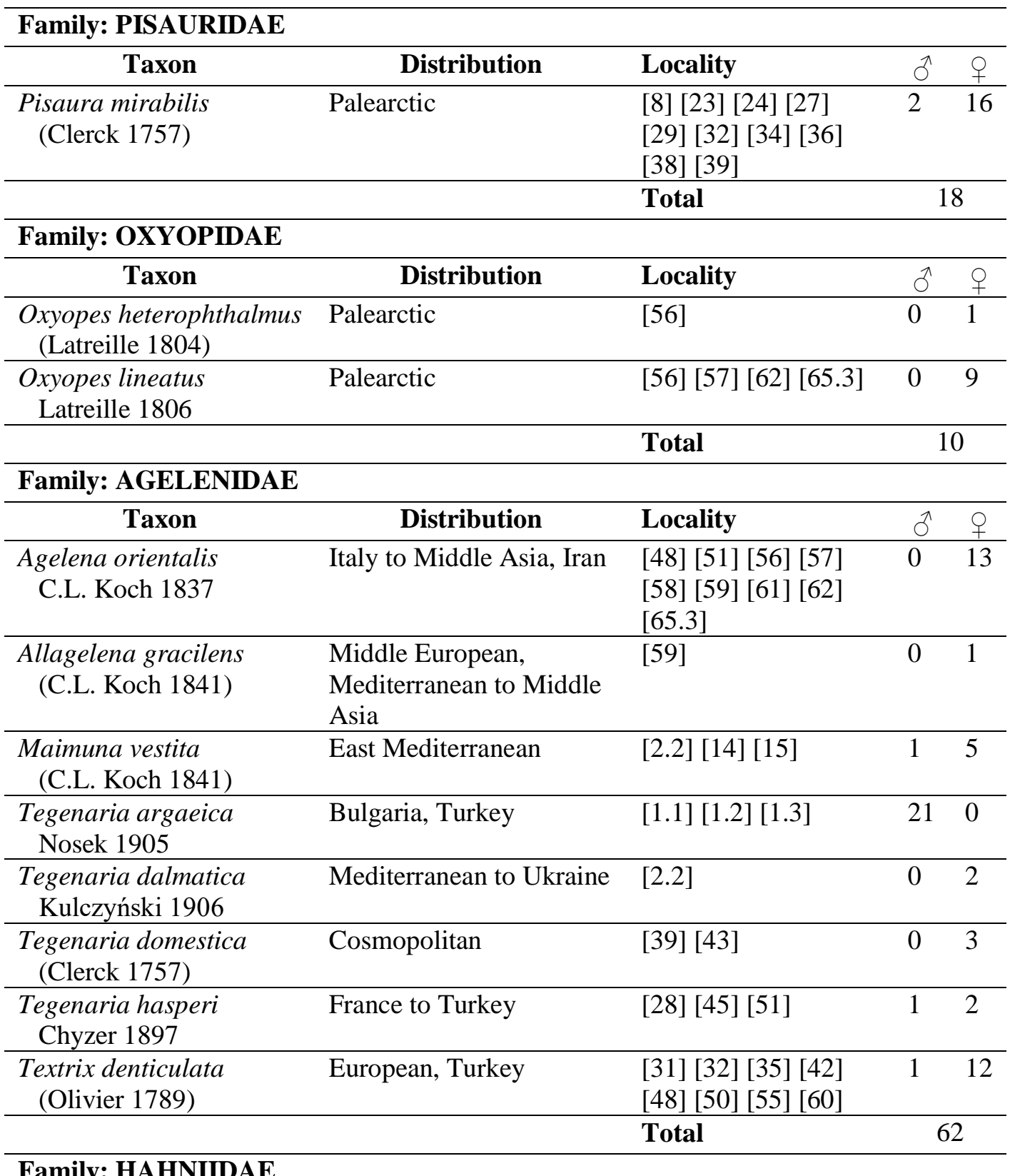

\begin{tabular}{|c|c|c|c|c|}
\hline Taxon & Distribution & Locality & 0 & o \\
\hline $\begin{array}{l}\text { Hahnia nava } \\
\quad \text { (Blackwall 1841) }\end{array}$ & Palearctic & {$[38]$} & 0 & 1 \\
\hline \multirow[t]{2}{*}{$\begin{array}{l}\text { Iberina montana } \\
\text { (Blackwall 1841) }\end{array}$} & European, Turkey, Russia & $\begin{array}{l}{[1.2][1.3][1.6][34]} \\
{[35][48][55]}\end{array}$ & 3 & 5 \\
\hline & & Total & & \\
\hline
\end{tabular}

Family: DICTYNIDAE

\begin{tabular}{cllcc}
\hline \multicolumn{1}{c}{ Taxon } & \multicolumn{1}{c}{ Distribution } & Locality & $\delta^{\lambda}$ & 我 \\
\hline $\begin{array}{c}\text { Brigittea latens } \\
\text { (Fabricius 1775) }\end{array}$ & European to Middle Asia & {$[16][23][32][34]$} & 8 & 4 \\
\hline $\begin{array}{c}\text { Dictyna arundinacea } \\
\text { (Linnaeus 1758) }\end{array}$ & Holarctic & {$[35][44]$} & & \\
\hline
\end{tabular}


Table 2 (continued)

\begin{tabular}{|c|c|c|c|}
\hline $\begin{array}{l}\text { Dictyna uncinata } \\
\text { Thorell } 1856\end{array}$ & Palearctic & $\begin{array}{l}{[21][23][25][26]} \\
{[30][31][33][35]}\end{array}$ & 6 \\
\hline $\begin{array}{l}\text { Lathys humilis } \\
\text { (Blackwall 1855) }\end{array}$ & Palearctic & $\begin{array}{l}{[21][23][28][30]} \\
{[38][40][42][43]} \\
{[44]}\end{array}$ & 26 \\
\hline $\begin{array}{l}\text { Lathys lehtineni } \\
\text { Kovblyuk, Kastrygina } \\
\text { and Omelko } 2014\end{array}$ & Ukraine, Turkey & {$[16][37]$} & $\begin{array}{ll}0 & 12\end{array}$ \\
\hline $\begin{array}{l}\text { Nigma flavescens } \\
\text { (Walckenaer 1830) }\end{array}$ & European & $\begin{array}{l}{[20][21][26][30]} \\
{[33][35][39][42]} \\
{[44]}\end{array}$ & $25 \quad 25$ \\
\hline & & Total & 124 \\
\hline $\begin{array}{l}\text { Family: } \\
\text { AMAUROBIIDAE }\end{array}$ & & & \\
\hline Taxon & Distribution & Locality & $\hat{0}$ \\
\hline $\begin{array}{l}\text { Amaurobius erberi } \\
\text { (Keyserling 1863) }\end{array}$ & $\begin{array}{l}\text { European, Canarian } \\
\text { Island }\end{array}$ & $\begin{array}{l}{[1.4][1.1][1.2][2.1]} \\
{[2.2][10][16][17]} \\
{[21][26][27][30]} \\
{[34][36][38][43]} \\
{[44][55][65.3]} \\
{[65.2]}\end{array}$ & 14 \\
\hline
\end{tabular}

Family:

TITANOECIDAE

\begin{tabular}{cllcc}
\hline Taxon & \multicolumn{1}{c}{ Distribution } & Locality & 0 & + \\
\hline $\begin{array}{c}\text { Nurscia albomaculata } \\
\text { (Lucas 1846) }\end{array}$ & $\begin{array}{l}\text { European, Turkey, Egypt } \\
\text { to Middle Asia }\end{array}$ & {$[13.8]$} & 2 & 0 \\
\hline & & Total & 2
\end{tabular}

Family:

EUTICHURIDAE

\begin{tabular}{|c|c|c|c|}
\hline Taxon & Distribution & Locality & $\hat{0}$ \\
\hline $\begin{array}{l}\text { Cheiracanthium elegans } \\
\text { Thorell } 1875\end{array}$ & European to Middle Asia & {$[13.3]$} & 1 \\
\hline $\begin{array}{l}\text { Cheiracanthium } \\
\text { erraticum } \\
\text { (Walckenaer 1802) }\end{array}$ & Palearctic & [38] & 0 \\
\hline \multirow[t]{2}{*}{$\begin{array}{l}\text { Cheiracanthium mildei } \\
\text { L. Koch } 1864\end{array}$} & Holarctic, Argentina & $\begin{array}{l}{[23][29][34][37]} \\
{[39]}\end{array}$ & 4 \\
\hline & & Total & 10 \\
\hline \multicolumn{4}{|l|}{ Family: MITURGIDAE } \\
\hline Taxon & Distribution & Locality & $\hat{0}$ \\
\hline \multirow[t]{2}{*}{$\begin{array}{l}\text { Zora spinimana } \\
\quad \text { (Sundevall 1833) }\end{array}$} & Palearctic & $\begin{array}{l}{[20][21][25][35]} \\
{[39][44]}\end{array}$ & 3 \\
\hline & & Total & 11 \\
\hline
\end{tabular}


Table 2 (continued)

Family:

ANYPHAENIDAE

\begin{tabular}{|c|c|c|c|}
\hline Taxon & Distribution & Locality & $\hat{0}$ \\
\hline $\begin{array}{l}\text { Anyphaena accentuata } \\
\text { (Walckenaer 1802) }\end{array}$ & $\begin{array}{l}\text { European to Middle Asia, } \\
\text { Iran }\end{array}$ & [24] [36] [38] & 2 \\
\hline $\begin{array}{l}\text { Anyphaena sabina } \\
\text { L. Koch } 1866\end{array}$ & $\begin{array}{l}\text { European, Turkey, } \\
\text { Russia, Georgia, } \\
\text { Azerbaijan }\end{array}$ & [24] [34] [36] & 5 \\
\hline
\end{tabular}

Family:

LIOCRANIDAE

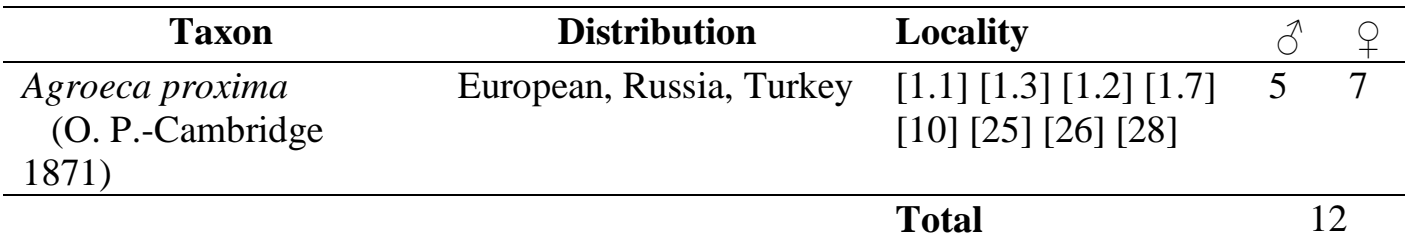

Family:

PHRUROLITHIDAE

\begin{tabular}{|c|c|c|c|c|}
\hline \multirow{2}{*}{$\begin{array}{c}\text { Taxon } \\
\text { Phrurolithus festivus } \\
\text { (C.L. Koch 1835) }\end{array}$} & Distribution & Locality & र & q \\
\hline & Palearctic & {$[24][32][50][65.2]$} & 7 & 1 \\
\hline & & Total & & 8 \\
\hline
\end{tabular}

Family:

CLUBIONIDAE

\begin{tabular}{cllcc}
\hline \multicolumn{1}{c}{ Taxon } & \multicolumn{1}{c}{ Distribution } & Locality & \multirow{2}{c}{} & \multicolumn{1}{c}{} \\
\hline Clubiona comta & European, Russia, North & {$[21][23][24][27]$} & 2 & 19 \\
C.L. Koch 1839 & Africa & {$[28][30][33][35]$} & & \\
& & {$[39][43]$} & & \\
\hline Clubiona neglecta & Palearctic & {$[43]$} & 0 & 1
\end{tabular}

O. P.-Cambridge 1862

Total 22

Family: ZODARIIDAE

\begin{tabular}{|c|c|c|c|c|}
\hline Taxon & Distribution & Locality & 0 & q \\
\hline \multirow[t]{2}{*}{$\begin{array}{c}\text { Zodarion abantense } \\
\text { Wunderlich } 1980 \\
\end{array}$} & Turkey, Russia, Georgia & {$[27][30][60]$} & 2 & 4 \\
\hline & & Total & & 5 \\
\hline \multicolumn{5}{|l|}{$\begin{array}{l}\text { Family: } \\
\text { GNAPHOSIDAE }\end{array}$} \\
\hline Taxon & Distribution & Locality & 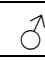 & q \\
\hline $\begin{array}{l}\text { Aphantaulax cincta } \\
\text { (L. Koch 1866) }\end{array}$ & $\begin{array}{l}\text { European, Turkey, North } \\
\text { Africa, Israel }\end{array}$ & [29] [23] & 4 & 0 \\
\hline $\begin{array}{r}\text { Callilepis cretica } \\
\text { (Roewer 1928) }\end{array}$ & $\begin{array}{l}\text { Macedonia, Greece, Crete, } \\
\text { Turkey, Azerbaijan }\end{array}$ & [56] & 0 & 1 \\
\hline $\begin{array}{l}\text { Callilepis nocturna } \\
\text { (Linnaeus 1758) }\end{array}$ & Palearctic & [4] [6] & 0 & 3 \\
\hline $\begin{array}{r}\text { Drassodes lapidosus } \\
\text { (Walckenaer 1802) }\end{array}$ & Palearctic & $\begin{array}{l}{[4][16][24][32][34]} \\
{[36][37][38][39]} \\
{[41][42][43]}\end{array}$ & 21 & 20 \\
\hline
\end{tabular}


Table 2 (continued)

\begin{tabular}{|c|c|c|c|}
\hline $\begin{array}{l}\text { Drassodes pubescens } \\
\text { (Thorell 1856) }\end{array}$ & Palearctic & [32] & 10 \\
\hline $\begin{array}{l}\text { Drassyllus praeficus } \\
\text { (L. Koch 1866) }\end{array}$ & European to Middle Asia & [34] [38] & 3 \\
\hline $\begin{array}{l}\text { Gnaphosa lucifuga } \\
\text { (Walckenaer 1802) }\end{array}$ & Palearctic & [4] [6] [16] [32] & 11 \\
\hline $\begin{array}{l}\text { Gnaphosa opaca } \\
\text { Herman } 1879\end{array}$ & European to Middle Asia & [3] & 1 \\
\hline $\begin{array}{l}\text { Haplodrassus } \\
\text { dalmatensis } \\
\text { (L. Koch 1866) }\end{array}$ & Palearctic, Ethiopia & [4] & 0 \\
\hline $\begin{array}{l}\text { Haplodrassus minor } \\
\text { (O. P.-Cambridge } \\
\text { 1879) }\end{array}$ & $\begin{array}{l}\text { European, Ukraine, } \\
\text { Russia }\end{array}$ & [39] [41] & 0 \\
\hline $\begin{array}{l}\text { Haplodrassus signifer } \\
\text { (C.L. Koch 1839) }\end{array}$ & Holarctic & [3] [4] [16] [41] & 6 \\
\hline $\begin{array}{l}\text { Micaria albovittata } \\
\text { (Lucas 1846) }\end{array}$ & Palearctic & [4] & 2 \\
\hline $\begin{array}{l}\text { Micaria dives (Lucas } \\
1846 \text { ) }\end{array}$ & Palearctic & [4] & 0 \\
\hline $\begin{array}{l}\text { Nomisia aussereri } \\
\text { (L. Koch 1872) }\end{array}$ & Palearctic & {$[64][65.3]$} & 3 \\
\hline $\begin{array}{l}\text { Nomisia exornata } \\
\text { (C.L. Koch 1839) }\end{array}$ & European to Middle Asia & [34] & 2 \\
\hline $\begin{array}{l}\text { Poecilochroa variana } \\
\text { (C.L. Koch 1839) }\end{array}$ & European to Middle Asia & [32] & 1 \\
\hline $\begin{array}{l}\text { Scotophaeus scutulatus } \\
\text { (L. Koch 1866) }\end{array}$ & $\begin{array}{l}\text { European to Middle Asia, } \\
\text { Algeria }\end{array}$ & [35] & 0 \\
\hline $\begin{array}{l}\text { Trachyzelotes pedestris } \\
\text { (C.L. Koch 1837) }\end{array}$ & European to Iran & [39] [41] & 2 \\
\hline $\begin{array}{l}\text { Zelotes apricorum } \\
\text { (L. Koch 1876) }\end{array}$ & European to Kazakhstan & [26] [55] & 0 \\
\hline $\begin{array}{l}\text { Zelotes strandi } \\
\text { (Nosek 1905) }\end{array}$ & Bulgaria, Turkey & $\begin{array}{l}1.1][16][32][34] \\
{[36][37][42][65.3]}\end{array}$ & 20 \\
\hline $\begin{array}{l}\text { Zelotes tenuis } \\
\text { (L. Koch 1866) }\end{array}$ & $\begin{array}{l}\text { Mediterranean to Russia, } \\
\text { Iran, USA }\end{array}$ & [45] & 0 \\
\hline & & Total & 120 \\
\hline \multicolumn{4}{|l|}{$\begin{array}{l}\text { Family: } \\
\text { SPARASSIDAE }\end{array}$} \\
\hline Taxon & Distribution & Locality & $\hat{0}$ \\
\hline \multirow[t]{2}{*}{$\begin{array}{l}\text { Micrommata virescens } \\
\text { (Clerck 1757) }\end{array}$} & Palearctic & {$[23][25]$} & 0 \\
\hline & & Total & 2 \\
\hline \multicolumn{4}{|l|}{$\begin{array}{l}\text { Family: } \\
\text { PHILODROMIDAE }\end{array}$} \\
\hline Taxon & Distribution & Locality & $\hat{0}$ \\
\hline $\begin{array}{l}\text { Philodromus aureoles } \\
\text { (Clerck 1757) }\end{array}$ & European & [48] [55] [62] & 0 \\
\hline
\end{tabular}


Table 2 (continued)

\begin{tabular}{lllll}
\hline $\begin{array}{c}\text { Philodromus dispar } \\
\text { Walckenaer 1826 }\end{array}$ & European to Middle Asia & $\begin{array}{l}{[21][27][38][41]} \\
{[44]}\end{array}$ & 0 & 8 \\
\hline $\begin{array}{l}\text { Philodromus krausi } \\
\text { Muster and Thaler 2004 }\end{array}$ & Greece, Turkey & {$[40]$} & 0 & 1 \\
\hline $\begin{array}{l}\text { Philodromus rufus } \\
\text { Walckenaer 1826 }\end{array}$ & Holarctic & {$[25][27][29][33]$} & 2 & 8 \\
& & {$[36][37][38][44]$} & & \\
\hline $\begin{array}{l}\text { Pulchellodromus } \\
\text { pulchellus } \\
\text { (Lucas 1846) }\end{array}$ & Mediterranean & {$[65.2]$} & 1 & 0 \\
\hline
\end{tabular}

\begin{tabular}{|c|c|c|c|c|}
\hline & & Total & 2 & \\
\hline Family: THOMISIDAE & & & & \\
\hline Taxon & Distribution & Locality & $\hat{0}$ & q \\
\hline $\begin{array}{l}\text { Cozyptila thaleri } \\
\text { Marusik and Kovblyuk } \\
2005\end{array}$ & Greece, Turkey, Ukraine & [42] & 0 & 1 \\
\hline $\begin{array}{l}\text { Diaea dorsata } \\
\text { (Fabricius 1777) }\end{array}$ & Palearctic & [27] [37] & 1 & 1 \\
\hline $\begin{array}{l}\text { Misumena vatia } \\
\text { (Clerck 1757) }\end{array}$ & Holarctic & $\begin{array}{l}{[9][23][24][25]} \\
{[26][29][37][42]} \\
{[44][45][65.2]}\end{array}$ & 10 & 8 \\
\hline $\begin{array}{l}\text { Ozyptila praticola } \\
\text { (C.L. Koch 1837) }\end{array}$ & Holarctic & [42] & 0 & 1 \\
\hline $\begin{array}{l}\text { Ozyptila sanctuaria } \\
\text { (O. P.-Cambridge }\end{array}$ & European & [1.1] [65.3] & 6 & 0 \\
\hline
\end{tabular}
1871)

\begin{tabular}{|c|c|c|c|}
\hline $\begin{array}{l}\text { Pistius truncatus } \\
\text { (Pallas 1772) }\end{array}$ & Palearctic & [29] & 1 \\
\hline $\begin{array}{r}\text { Runcinia grammica } \\
\text { (C.L. Koch 1837) }\end{array}$ & $\begin{array}{l}\text { Palearctic, St. Helena, } \\
\text { South Africa, Lesotho }\end{array}$ & [45] & 2 \\
\hline $\begin{array}{l}\text { Synema globosum } \\
\text { (Fabricius 1775) }\end{array}$ & Palearctic & [9] [65.2] & 1 \\
\hline $\begin{array}{l}\text { Thomisus onustus } \\
\text { Walckenaer } 1805\end{array}$ & Palearctic & [23] [28] [34] [65.2] & 4 \\
\hline $\begin{array}{l}\text { Tmarus piger } \\
\text { (Walckenaer 1802) }\end{array}$ & Palearctic & [8] & 1 \\
\hline $\begin{array}{l}\text { Xysticus acerbus } \\
\text { Thorell } 1872\end{array}$ & European to Middle Asia & [44] & 0 \\
\hline $\begin{array}{l}\text { Xysticus audax } \\
\text { (Schrank 1803) }\end{array}$ & Palearctic & [44] & 0 \\
\hline $\begin{array}{l}\text { Xysticus cristatus } \\
\text { (Clerck 1757) }\end{array}$ & Palearctic & $\begin{array}{l}{[28][32][33][37]} \\
{[44]}\end{array}$ & 0 \\
\hline $\begin{array}{l}\text { Xysticus kochi } \\
\text { Thorell } 1872 \\
\end{array}$ & $\begin{array}{l}\text { European, Mediterranean } \\
\text { to Middle Asia }\end{array}$ & $\begin{array}{l}{[4][23][27][28]} \\
{[32][37]}\end{array}$ & 4 \\
\hline $\begin{array}{l}\text { Xysticus lanio } \\
\text { C.L. Koch } 1835\end{array}$ & Palearctic & $\begin{array}{l}{[21][23][29][38]} \\
{[39][44]}\end{array}$ & 6 \\
\hline \multirow[t]{2}{*}{$\begin{array}{l}\text { Xysticus striatipes } \\
\text { L. Koch } 1870\end{array}$} & Palearctic & {$[32]$} & 0 \\
\hline & & Total & 77 \\
\hline
\end{tabular}


Table 2 (continued)

\begin{tabular}{|c|c|c|c|c|}
\hline Family: SALTICIDAE & & & & \\
\hline $\begin{array}{c}\text { Taxon } \\
\end{array}$ & Distribution & Locality & $\hat{0}$ & q \\
\hline $\begin{array}{l}\text { Aelurillus } v \text {-insignitus } \\
\text { (Clerck 1757) }\end{array}$ & Palearctic & [37] [15] [14] [28] & 5 & 1 \\
\hline $\begin{array}{l}\text { Ballus chalybeius } \\
\text { (Walckenaer 1802) }\end{array}$ & $\begin{array}{l}\text { European, North Africa } \\
\text { to Middle Asia }\end{array}$ & $\begin{array}{l}26][27][28][29] \\
{[33][35][38][65.2]}\end{array}$ & 3 & 9 \\
\hline $\begin{array}{l}\text { Carrhotus xanthogramma } \\
\text { (Latreille 1819) }\end{array}$ & Palearctic & [23] [26] [29] [65.2] & 5 & 6 \\
\hline $\begin{array}{l}\text { Chalcoscirtus infimus } \\
\text { (Simon 1868) }\end{array}$ & $\begin{array}{l}\text { South, Middle European } \\
\text { to Middle Asia }\end{array}$ & {$[37]$} & 2 & 1 \\
\hline $\begin{array}{l}\text { Euophrys frontalis } \\
\text { (Walckenaer 1802) }\end{array}$ & Palearctic & $\begin{array}{l}{[6][16][32][38]} \\
{[37][43]}\end{array}$ & 6 & 1 \\
\hline $\begin{array}{l}\text { Euophrys herbigrada } \\
\text { (Simon 1871) }\end{array}$ & Europe & [26] [30] [38] & 5 & 7 \\
\hline $\begin{array}{l}\text { Evarcha arcuata } \\
\text { (Clerck 1757) }\end{array}$ & Palearctic & $\begin{array}{l}{[13.8][25][29][34]} \\
{[37][38][47][65.2]} \\
{[65.3]}\end{array}$ & 1 & 15 \\
\hline $\begin{array}{r}\text { Evarcha falcata } \\
\text { (Clerck 1757) }\end{array}$ & Palearctic & $\begin{array}{l}{[20][21][23][29]} \\
{[30][38][39][61]}\end{array}$ & 11 & 1 \\
\hline $\begin{array}{l}\text { Heliophanus auratus } \\
\text { C.L. Koch } 1835 \\
\end{array}$ & Palearctic & {$[16][42][47]$} & 1 & 2 \\
\hline $\begin{array}{r}\text { Heliophanus cupreus } \\
\text { (Walckenaer 1802) }\end{array}$ & Palearctic & $\begin{array}{l}{[26][28][29][33]} \\
{[34][35][36][37]} \\
{[38][39][42][62]} \\
{[65.2]}\end{array}$ & 16 & 13 \\
\hline $\begin{array}{l}\text { Heliophanus flavipes } \\
\text { (Hahn 1832) }\end{array}$ & Palearctic & {$[4][36][65.2]$} & 3 & 2 \\
\hline $\begin{array}{l}\text { Heliophanus kochii } \\
\text { Simon } 1868 \\
\end{array}$ & Palearctic, USA & $\begin{array}{l}{[28][32][34][37]} \\
{[57]}\end{array}$ & 5 & 4 \\
\hline $\begin{array}{l}\text { Heliophanus tribulosus } \\
\text { Simon } 1868\end{array}$ & European to Middle Asia & $\begin{array}{l}{[28][37][38][56]} \\
{[61]}\end{array}$ & 4 & 3 \\
\hline $\begin{array}{l}\text { Macaroeris nidicolens } \\
\text { (Walckenaer 1802) }\end{array}$ & $\begin{array}{l}\text { Portugal to Cyprus, } \\
\text { Turkey, Sri Lanka }\end{array}$ & $\begin{array}{l}{[23][25][37][38]} \\
{[48][56][58][62]} \\
{[65.2]}\end{array}$ & 3 & 9 \\
\hline $\begin{array}{l}\text { Marpissa muscosa } \\
\text { (Clerck 1757) }\end{array}$ & Palearctic & {$[21]$} & 0 & 1 \\
\hline Neon levis (Simon 1871) & Palearctic & [26] & 1 & 0 \\
\hline $\begin{array}{l}\text { Neon reticulatus } \\
\text { (Blackwall 1853) }\end{array}$ & Holarctic & {$[40][51]$} & 0 & 3 \\
\hline $\begin{array}{l}\text { Pellenes nigrociliatus } \\
\text { (Simon 1875) }\end{array}$ & Palearctic & {$[65.2]$} & 0 & 1 \\
\hline $\begin{array}{l}\text { Philaeus chrysops } \\
\text { (Poda 1761) }\end{array}$ & Palearctic & [6] [34] [37] [39] & 6 & 3 \\
\hline $\begin{array}{l}\text { Phintella castriesiana } \\
\text { (Grube 1861) }\end{array}$ & Palearctic & [23] [24] [25] [29] & 2 & 3 \\
\hline $\begin{array}{l}\text { Phlegra fasciata } \\
\text { (Hahn 1826) }\end{array}$ & Palearctic & $\begin{array}{l}28][32][34][37] \\
{[39][41][47][65.2]}\end{array}$ & 6 & 9 \\
\hline $\begin{array}{l}\text { Pseudeuophrys erratica } \\
\text { (Walckenaer 1826) }\end{array}$ & Palearctic & $\begin{array}{l}\text { [21] [25] [26] [31] } \\
{[49]}\end{array}$ & 4 & 1 \\
\hline
\end{tabular}


Table 2 (continued)

\begin{tabular}{lllll}
\hline $\begin{array}{c}\text { Pseudeuophrys obsoleta } \\
\text { (Simon 1868) }\end{array}$ & Palearctic & $\begin{array}{l}{[3][4][16][19][34]} \\
{[37][38][41]}\end{array}$ & 3 & 17 \\
\hline $\begin{array}{c}\text { Pseudicius picaceus } \\
\text { (Simon 1868) }\end{array}$ & $\begin{array}{l}\text { Mediterranean to } \\
\text { Azerbaijan }\end{array}$ & {$[37]$} & 1 & 0 \\
\hline $\begin{array}{c}\text { Salticus noordami } \\
\text { Metzner 1999 }\end{array}$ & $\begin{array}{l}\text { Greece, Turkey, Israel, } \\
\text { Iran }\end{array}$ & {$[62]$} & 0 & 1 \\
\hline $\begin{array}{c}\text { Sittipub pubescens } \\
\text { (Fabricius, 1775) }\end{array}$ & $\begin{array}{l}\text { European, Morocco, } \\
\text { Turkey, Russia }\end{array}$ & {$[42]$} & 1 & 0 \\
\hline $\begin{array}{c}\text { Synageles dalmaticus } \\
\text { (Keyserling 1863) }\end{array}$ & Mediterranean & {$[45]$} & 0 & 1 \\
\hline $\begin{array}{c}\text { Synageles hilarulus } \\
\text { (C.L. Koch 1846) }\end{array}$ & Palearctic & {$[59]$} & 0 & 1 \\
\hline $\begin{array}{c}\text { Talavera aequipes } \\
\text { (O. P.-Cambridge, }\end{array}$ & Palearctic & {$[34][38][43][47]$} & 0 & 9 \\
1871) & & Total & 218 \\
\hline
\end{tabular}

*New record for Turkey

A total of 1766 adult (627 male and 1139 female) spiders were collected, representing 200 species belonging to 133 genera in 31 families; $35.50 \%$ of the spiders collected were male and $64.50 \%$ female. Most of the species in the study area are Palearctic in terms of their distribution. During the field trips, only some male or female individuals were found for some of the species obtained from the research area. The most species-rich spider families are the Theridiidae (29 spp.) and the Salticidae (29 spp.) followed by the Gnaphosidae (21 spp.), Araneidae (20 spp.), Linyphiidae (20 spp.), and Thomisidae (16 spp.) (Table 2). The most abundant family in the field was the Araneidae (14\%), followed by the Linyphiidae (13\%), Theriididae (12\%), Salticidae (12\%), Lycosidae (8\%), Gnaphosidae (7\%), and Dictynidae (7\%) (Figure 2). Sancak (2007) reported that the most abundant family was the Lycosidae, followed by the Araneidae, Theriididae, Linyphiidae and Salticidae from the Eastern Black Sea Region. All the genera of the families Amaurobiidae, Liocranidae, Miturgidae, Oecobiidae, Phrurolithidae, Scytodidae, Sparassidae, Titanoecidae and
Zodariidae found were represented by one species each (Table 2).

The prevalence of the Araneidae, Linyphiidae and Theriididae in the samples is probably due to the sampling method used. Many of our collected grass-dwelling and ground-dwelling spiders (Araneidae, Linyphiidae and Theridiidae) are usually associated with low vegetation. The grassdwelling and ground-dwelling spider species were mostly obtained using both methods (sweep-netting and beating sheet). Besides, since the sweep-netting in our study was carried out only during the daytime, it was restricted to diurnal spider species (e.g. families Pisauridae, Salticidae and Oxyopidae), while the pitfall traps collected both diurnally active spiders (Lycosidae and Zoridae) and nocturnally active ones (Clubionidae, Gnaphosidae and Liocranidae). The ground-dwelling spiders were collected using pitfall traps; trapping was continuous, with traps being kept open from 2009 to 2012. For this reason, this study is poor in terms of nocturnal species and grounddwelling spiders. 


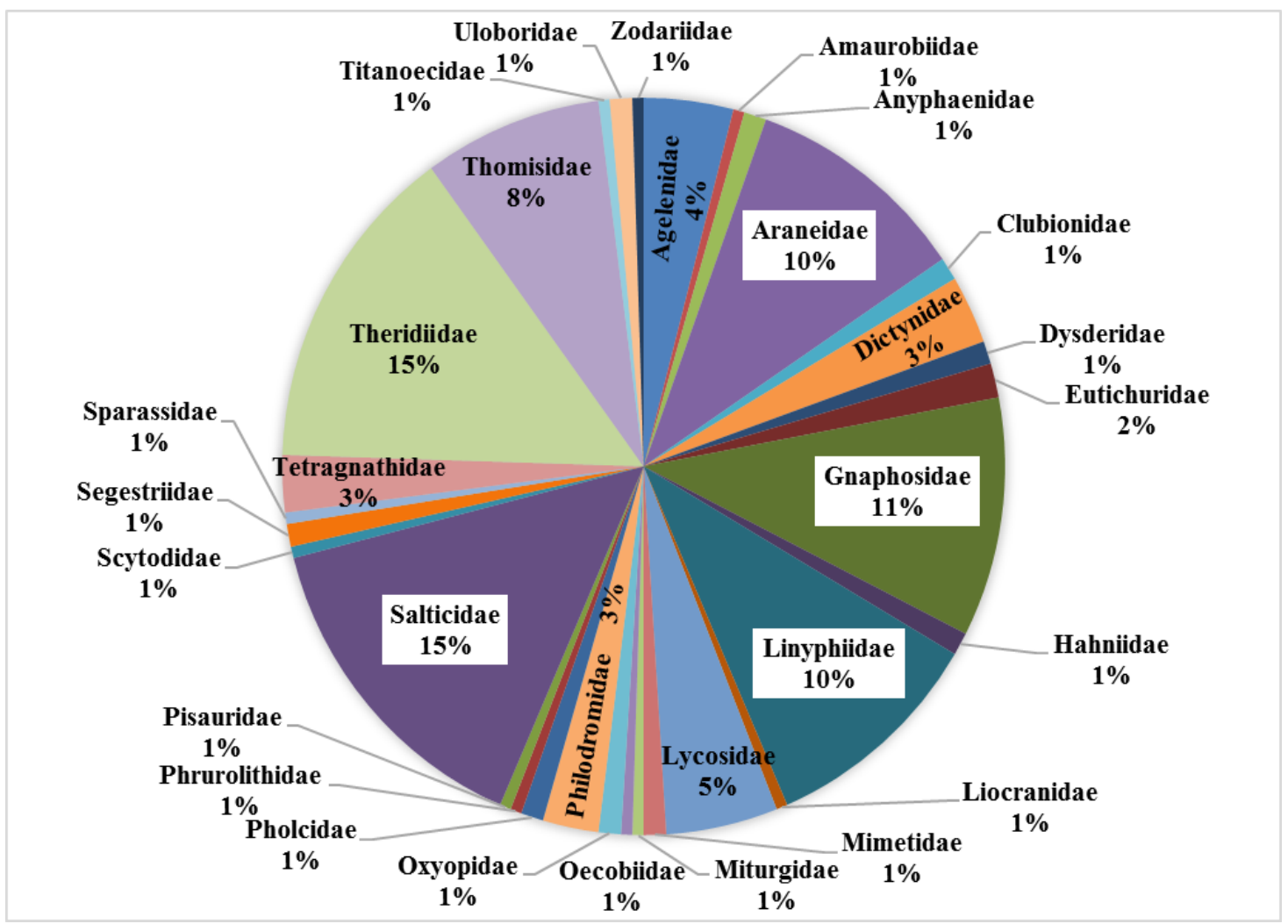

Figure 2. Percentage of species richness per family of spiders recorded in Sinop

Frontinellina frutetorum (C.L. Koch 1834), Anelosimus vittatus (C. L. Koch 1836), Mangora acaylpha (Walckenaer 1802) and Amaurobius erberi (Keyserling 1863) are the most common species in different localities, while the following species were found in a few localities, but they were sampled in higher numbers in the same localities: Zilla diodia (Walckenaer 1802) and Nigma flavescens (Walckenaer 1830).

In a faunistic study on the Linyphiidae family in the Black Sea Region, 14 genera and 17 species were recorded (Karabulut, 2011; Karabulut and Türkeş, 2011). In our study, 18 genera and 22 species belonging to the Linyphiidae family were found. The following species are common to both studies: Linyphia hortensis Sundevall 1830, Linyphia triangularis (Clerck 1757), Centromerus sylvaticus (Blackwall 1841), Neriene peltata (Wider 1834), Neriene radiata (Walckenaer, 1841), Frontinellina frutetorum (C.L. Koch 1834) and Lepthyphantes leprosus (Ohlert 1865).

The following spider species collected from the study area are known venomous spiders: Argiope lobata (Pallas 1772), Larinioides cornutus (Clerck 1757), Araneus diadematus Clerck 1757 belonging to family Araneidae; Cheiracanthium mildei (C.L. Koch 1864) to family Eutichuridae; Segestria florentina (Rossi 1790) to family Segestridae; Steatoda grossa (C.L. Koch 1838) and Steatoda paykulliana (Walckenaer $1805)$ to family Theridiidae (Bayram et al., 2007). Some species, namely, Alopecosa taeniopus (Kulczyński 1895), Myrmarachne formicaria (De Geer 1778), Orthobula charitonovi (Mikhailov 1986), Cyclosa oculata (Walckenaer 1802), Cicurina paphlagoniae Brignoli 1978, Walckenaeria furcillata (Menge 1869) and Clubiona terrestris Westring 1851 Coelotes sinopensis (Danışman, Karanfil \& Coşar 2016), Pellenes seriatus (Thorell 1875), Saitis barbipes (Simon 1868), Walckenaeria cirriceps Thaler 1996 that were previously reported for Sinop province (Topçu, Demir, \& Seyyar, 2005; Buchar and Dolanský, 2011; Danışman et al., 2012a, 2012b, 2016; Danışman, 2014; Danışman and Coşar, 2016; Brignoli, 1978; Coşar and Varol, 2016) were not found during this study (Table 3). In 
addition, Brignoli (1978), Coşar (2015), Danışman, Coşar, Erdek \& Sancak (2012d), Logunov and Demir (2006), Özkütük et al., (2015) and Coşar et al., (2017) reported species such as Neoscona adianta (Walckenaer 1802) Mangora acalypha
(Walckenaer 1802), Xysticus kochii Thorell 1872, Pseudicius picaceus (Simon, 1868) Harpactea korgei Brignoli 1979 and Haplodrassus minor (O.P.-Cambridge 1879) that were also collected during the present study (Table 3).

Table 3. Spiders according to records published until 2018 and results of this study

\begin{tabular}{|c|c|c|}
\hline $\begin{array}{l}\text { Family } \\
\text { species }\end{array}$ & Previously Reported & $\begin{array}{l}\text { Present } \\
\text { Study }\end{array}$ \\
\hline \multicolumn{3}{|l|}{ Family: Dysderidae } \\
\hline Harpactea korgei Brignoli, 1979 & Özkütük et al., 2015 & $\sqrt{ }$ \\
\hline \multicolumn{3}{|l|}{ Family: Linyphiidae } \\
\hline Walckenaeria furcillata (Menge, 1869) & Danışman 2014 & \\
\hline Walckenaeria cirriceps Thaler, 1996 & Danışman and Coşar 2016 & \\
\hline \multicolumn{3}{|l|}{ Family: Araneidae } \\
\hline Neoscona adianta (Walckenaer, 1802) & Brignoli 1978 & $\sqrt{ }$ \\
\hline Cyclosa oculata (Walckenaer 1802), & Brignoli 1978 & \\
\hline Mangora acalypha (Walckenaer, 1802) & Brignoli 1978 & $\sqrt{ }$ \\
\hline \multicolumn{3}{|l|}{ Family: Lycosidae } \\
\hline Alopecosa taeniopus (Kulczyński, 1895) & Buchar and Dolanský 2011 & \\
\hline \multicolumn{3}{|l|}{ Family: Agelenidae } \\
\hline Pireneitega cottarellii (Brignoli, 1978) & Brignoli 1978 & \\
\hline $\begin{array}{l}\text { Coelotes sinopensis Danışman, Karanfil \& } \\
\text { Coşar, } 2016\end{array}$ & Danışman et al. 2016 & \\
\hline
\end{tabular}

Family: Dictynidae

Cicurina paphlagoniae Brignoli, $1978 \quad$ Brignoli 1978

Family: Phrurolithidae

Orthobula charitonovi (Mikhailov, 1986) Danışman et al. 2012c

Family: Clubionidae

Clubiona terrestris Westring, 1851

Danışman et al. 2012b

Family: Gnaphosidae

Haplodrassus minor (O. P.-Cambridge, 1879) $\quad$ Coşar et al. $2017 \quad \sqrt{ }$

Family: Thomisidae

Xysticus kochi Thorell, 1872

Logunov and Demir 2006

Xysticus tenebrosus Silhavy, 1944

Coşar et al. 2016

Family: Salticidae

\begin{tabular}{llc}
\hline Pellenes seriatus (Thorell, 1875) & Coşar and Varol 2016 & \\
\hline Saitis barbipes (Simon, 1868) & Coşar and Varol 2016 & \\
\hline Pseudicius picaceus (Simon, 1868) & Danışman et al. 2012b, 2012a & $\sqrt{ }$ \\
\hline Neon levis (Simon, 1871) & Coşar 2015 & $\sqrt{ }$ \\
\hline
\end{tabular}

Regarding the new records for Turkey, one genus and three species have not been reported previously from the country. For Theridiidae, the genus Theridula Emerton
1882 and the species Asagena meridionalis Kulczyński 1894 (Figure 3), Theridion boesenbergi Strand 1904 (Figure 4) and 
Theridula gonygaster (Simon, 1873) (Figure 5 ) are reported for the first time from Turkey.

The current study provides the first detailed report on the spider richness of Sinop province. With the ecology and diversity of the spider fauna of the Black Sea
Region being so poorly known, each research effort contributes to our understanding of the geographical distribution of spider species. This study presents new distribution records for 192 species and indicates that spider diversity in Sinop province may be greater.

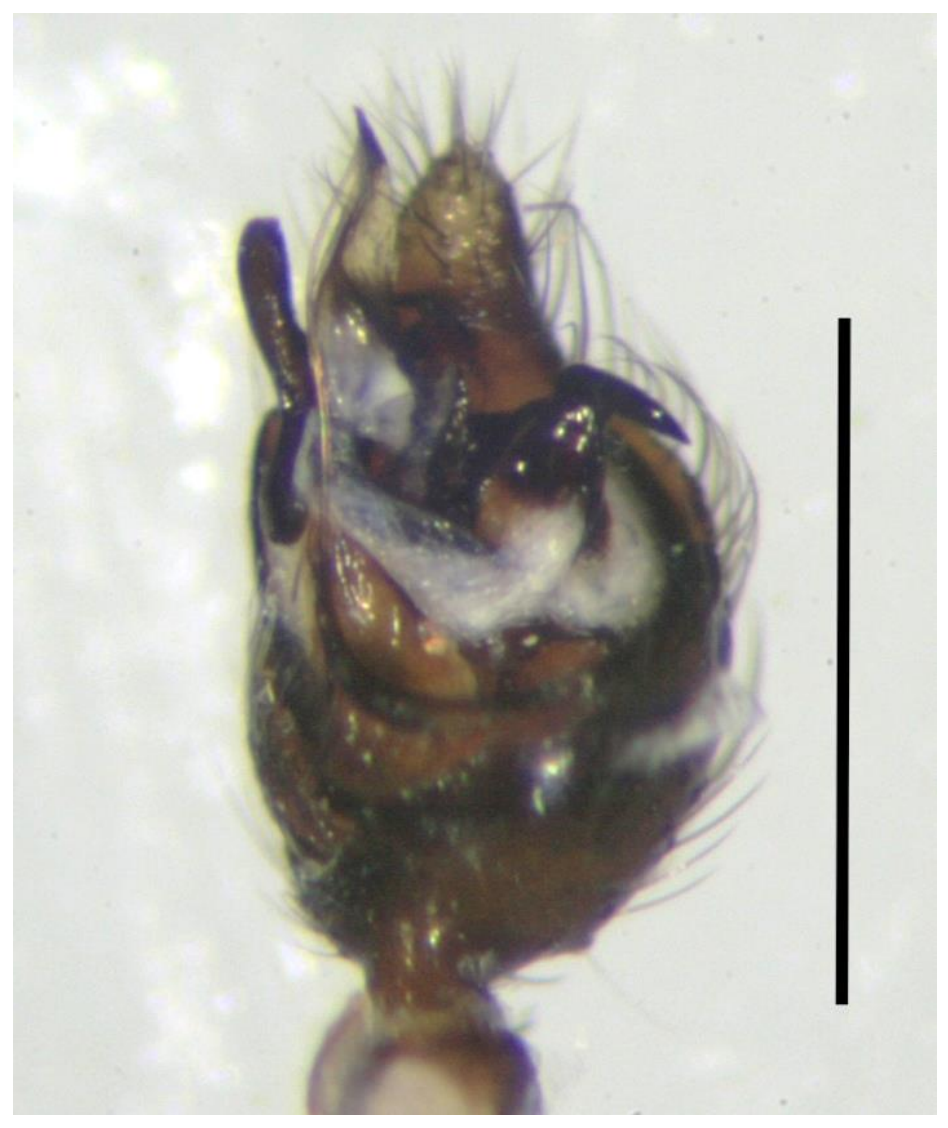

Figure 3. Asagena meridionalis Kulczyński 1894, male, pedipalp, ventral view. Scale bar $=1 \mathrm{~mm}$ 


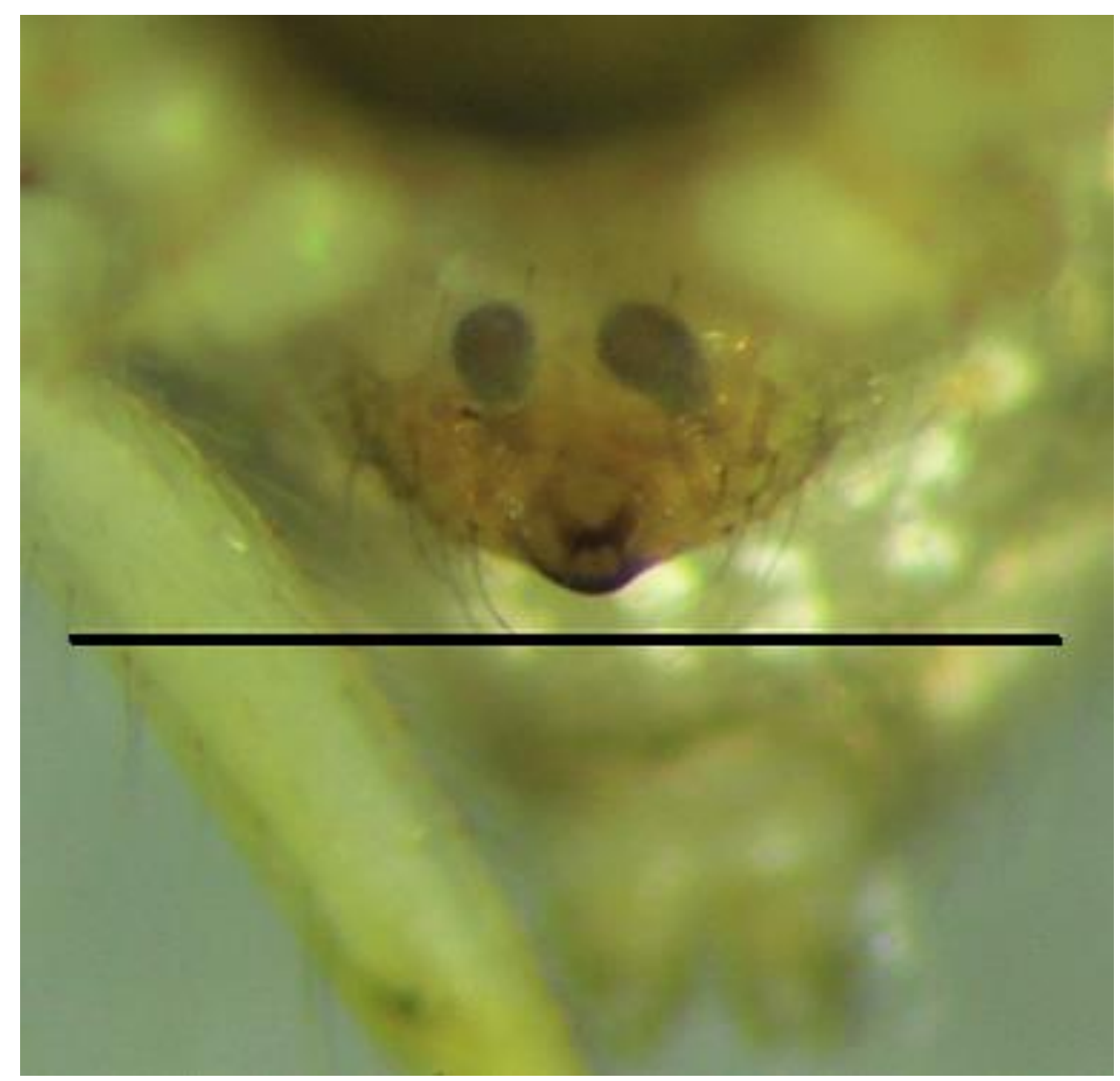

Figure 4. Theridion boesenbergi Strand 1904, female, ventral view of epigyne. Scale bar $=1 \mathrm{~mm}$. 


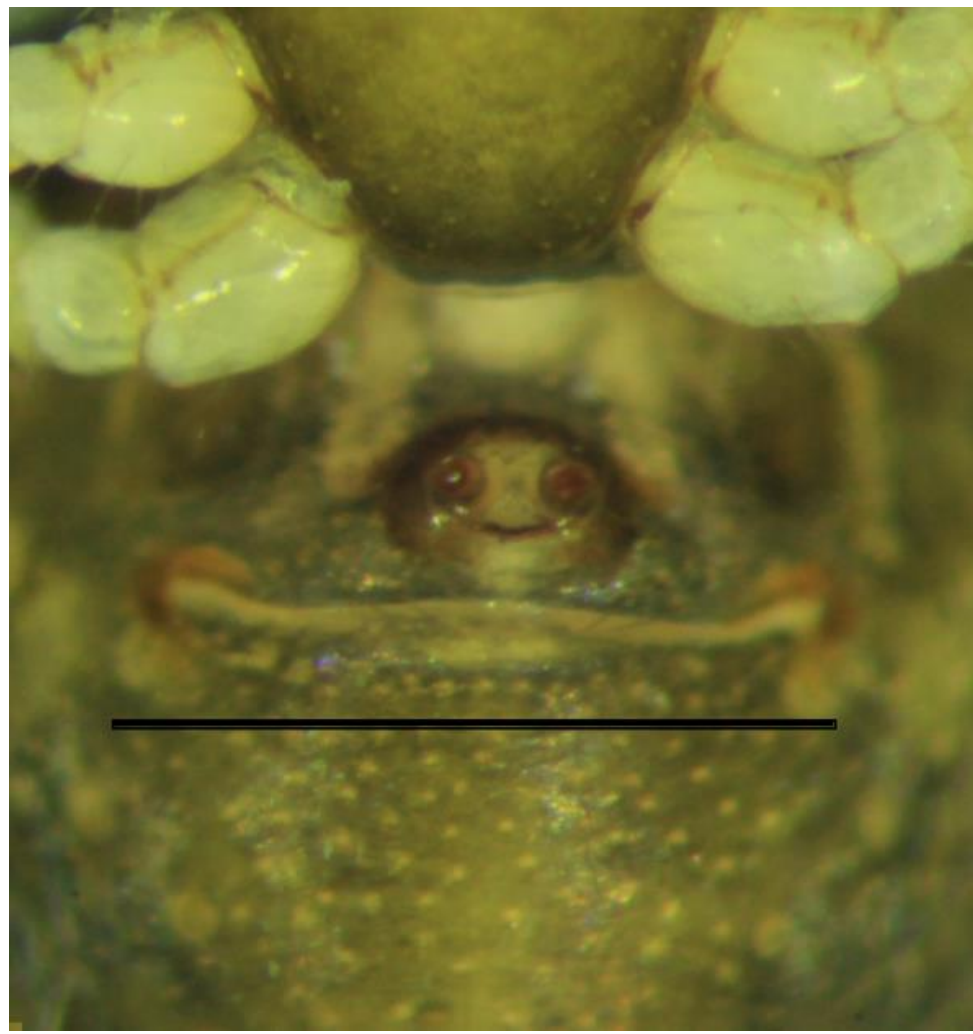

Figure 5. Theridula gonygaster (Simon 1873), female, ventral view of epigyne. Scale bar=1 mm.

\section{Acknowledgement}

This study was financially supported by Sinop University, Coordinatorship of Research Projects, with ID Number FEF1901-12-02.

\section{References}

Anonymous.

http://www.csb.gov.tr/turkce/dosya

(2012).

/ced/icdr2011/sinop icdr2011.pdf. 2011 Y11 Il Çevre Durum Raporu, Sinop. (accessed: 03.02.2015)

Bayram, A. (1987). Doğu Canik Dağlart Örümcekleri (Yüksek Lisans Tezi, MSc). Ankara Üniversitesi, Fen Bilimleri Enstitüsü, Ankara.

Bayram, A. (2002). Distributions of Turkish Spiders. In: Demirsoy, A., Ed. Zoogeography of Turkey. Meteksan Pub., Ankara, 1005p.

Bayram, A., Yiğit, N., Danışman, T., Çorak. İ., Sancak, Z. \& Ulaşoğlu, D. (2007). Venomous spiders of turkey (Araneae), Journal of Applied Biological Sciences, 1 (3), 33-36.

Bayram, A., Kunt, K.B. \& Danışman, T. (2017). The checklist of the spiders of Turkey. Version 2017, Online at http://www.spidersofturkey.info
Baysal, M. (2008). Çangal ormanı (SinopAyancık) vasküler bitkiler florası. Doktora Tezi, Ankara Üniversitesi, Ankara.

Brignoli, P.M. (1978). Ragni di Turchia V. Specie nuove o interessanti, cavernicole ed epigee, di varie famiglie (Araneae). Revue Suisse de Zoologie, 85, 461-541.

Buchar, J. \& Dolanský, J. (2011). New records of wolf spiders (Araneae: Lycosidae) in the Mediterranean. Klapalekiana, 47, 5-11.

Coşar, İ. (2015). Four new records for the spider fauna of Turkey (Araneae: Salticidae). Turkish Journal of Zoology, 39, 368-371.

Coşar, İ. \& Varol, İ. (2016). Six new records for the spider fauna of Turkey (Araneae: Salticidae) Turkish Journal of Entomology, 40(2), 157-163.

Coşar, İ., Danışman, T. \& Tarlabölen, R. (2016). A new crab spider record from Turkey (Araneae: Thomisidae). Serket, 15(2), 104106.

Coşar, İ., Danışman, T. \& Kartaler, M. (2017). Three new records for the spider fauna of Turkey (Araneae: Gnaphosidae). Indian Journal of Arachnology, 6(1), 34-38.

Danışman, T. (2014). First record of the linyphiid spider Walckenaeria furcillata (Menge, 1869) (Araneae, Linyphiidae) in Turkey. Serket, 14(2), 68-70. 
Danışman, T. \& Coşar, İ. (2016). Walckenaeria cirriceps Thaler, 1996, a new record from Turkey (Araneae: Linyphiidae). Serket, 15(1), 47-49.

Danışman, T., Coşar, İ., Erdek, M. \& Sancak, Z. (2012d). A contribution to the knowledge of Turkish jumping spider fauna (Araneae: Salticidae). Acta Zoologica Bulgarica, 64(2), 215-216.

Danışman, T., Erdek, M. \& Coşar, İ. (2012a). A new clubionid spider record from Turkey (Araneae: Clubionidae). Serket, 13(1/2), 108110.

Danışman, T., Erdek, M., Sancak, Z. \& Coşar, İ. (2012b). A new genus record for the corinnid sac spider fauna of Turkey (Araneae: Corinnidae). Munis Entomology and Zoology, 7(2), 1097-1100.

Danışman, T., Erdek, M., Sancak, Z. \& Coşar, İ. (2012c). A new genus record for the corinnid sac spider fauna of Turkey (Araneae: Corinnidae). Munis Entomology and Zoology, 7(2), 1097-1100.

Danışman, T., Karanfil, K. C. \& Coşar, İ. (2016). A new species of Coelotes Blackwall, 1841 (Araneae: Agelenidae) from Turkey. Turkish Journal of Zoology, 40, 147-150.

Demir, H. \& Seyyar, O. (2017). Annotated checklist of the spiders of Turkey. Munis Entomology and Zoology, 12(2), 433-469.

Demirsoy, A. (2002). Genel zoocoğrafya ve türkiye coğrafyast. Meteksan, Ankara, $1005 p p$.

Karabulut, H. (2011). Karadeniz Bölgesi Linyphiidae (Araneae) Familyast Üzerine Faunistik Çallşmalar. Yüksek Lisans Tezi, Niğde Üniversitesi, Niğde.

Karabulut, H. \& Türkeş, T. (2011). New records of Linyphiidae (Araneae) for Turkish araneofauna. Serket, 12, 117-123.

Karol, S. (1967). Türkiye Örümcekleri. I. Ön Liste 1-37 pp. Ankara Üniversitesi Basımevi. Ankara.

Logunov, D.V. \& Demir, H. (2006). Further faunistic notes on Cozyptila and Xysticus from Turkey (Araneae, Thomisidae). Arachnologische Mitteilungen, 31, 38-43.

Nentwig, W., Blick, T., Gloor, D., Hänggi, A. \& Kropf, C. (2017). Spiders of Europe. www.araneae.unibe.ch. Version 08.2017

Nyffeler, M., Benz, G. (1987). Spiders in natural pest control: a review. Journal of Applied Entomology, 103, 321-339.

Özkütük, R.S., Kunt, K.B., Karakaş, G. \& Danışman, T. (2015). Redescription of Harpactea korgei Brignoli, 1979 (Araneae: Dysderidae) with the first description of the female. Arthropods, 4(2), 32-37.
Öztürk, A. (2010). Sinop ilindeki armut genotiplerinin morfolojik, pomolojik ve moleküler karakterizasyonu. Doktora Tezi, Ondokuz Mayıs Üniversitesi, Samsun.

Sancak, Z. (2007). Doğu karadeniz bölgesi örümceklerinin sistematik ve faunistik açıdan incelenmesi. Yüksek Lisans Tezi, Kırıkkale Üniversitesi, Kırıkkale.

Topçu, A., Demir, H. \& Seyyar, O. (2005). A Checklist of the spiders of Turkey. Serket, 9(4), 109-140.

World Spider Catalog (2018). World spider catalog. Natural History Museum Bern, online at http://wsc.nmbe.ch, version 19.0, accessed on \{date of access $\}$. doi: $10.24436 / 2$ 